\title{
Grouping and Trapping of Evaporating Droplets in an Oscillating Gas Flow
}

\author{
David Katoshevski ${ }^{1}$, Tal Shakked ${ }^{1}$, Sergei S. Sazhin ${ }^{2}$, Cyril Crua ${ }^{2}$, \\ and Morgan R. Heikal ${ }^{2}$ \\ ${ }^{1}$ Department of Environmental Engineering, Ben-Gurion University of the Negev \\ Beer-Sheva 84105, Israel \\ ${ }^{2}$ School of Engineering, Faculty of Science and Engineering \\ The University of Brighton \\ Brighton BN2 4GJ, UK
}

$\underline{\text { Contact information }}$

Dr David Katoshevski

(Building \# 39), Ben-Gurion University of the Negev

Beer-Sheva 84105

Israel

Tel. +972-8-6479094

Fax. +972-8-6472983

e-mail: davidk@bgu.ac.il 


\section{Abstract}

A new approach to the analysis of droplet grouping in an oscillating gas flow is suggested. This is based on the investigation of droplet trajectories in the frame of reference moving with the phase velocity of the wave. Although the equations involved are relatively simple, the analysis shows distinctive characteristics of grouping and non-grouping cases. In the case of grouping, droplet trajectories converge to the points for which the ratio of flow velocity in this frame of reference and the amplitude of flow oscillations is less than 1, and the cosine of this ratio is positive. In the case of non-grouping, droplet trajectories in this frame of reference oscillate around the translational velocity close to the velocity of flow in the same frame of reference. The effect of the droplet size on the grouping pattern is investigated. It has been pointed out that for the smaller droplets much more stable grouping is observed. The effect of droplet evaporation is studied in the limiting case when the contribution of the heatup period can be ignored. It is shown that evaporation can lead to droplet grouping even in the case when the non-evaporating droplets are not grouped. This is related to the reduction in droplets diameter during the evaporation process. Coupling between gas and droplets is shown to decrease the grouping tendency. A qualitative agreement between predictions of the model and in-house experimental observations referring to Diesel engine sprays has been demonstrated.

Keywords: spray, grouping, trapping, evaporation, coupled solution. 


\section{Introduction}

It is well known that the interaction of non-evaporating and evaporating droplets with the ambient turbulent gas is a complicated process, accurate numerical modeling of which is a challenging task (Gouesbet and Berlemont, 1999; Loth, 2000; Gao and Mashayek, 2004). Direct numerical simulation (DNS) and large eddy simulation (LES) are widely used (Mashayek and Pandya, 2003). Application of these methods would allow us to predict a general picture of droplet and gas dynamics in some relatively simple geometries but the underlying physics of many processes would largely remain hidden, due to the complexity of these methods.

An alternative approach to investigating this process is based on the application of relatively simple models, enabling one to focus on specific aspects of the process, and clarify the underlying physics of the phenomenon. For example Katoshevski et al (2005) studied the process of grouping of non-evaporating particles in an oscillating gas flow based on a simple 1D model. The analysis was performed only in a stationary frame of reference, and the effects of particles on gas were ignored. The essence of the processes studied is schematically illustrated in Fig. 1. Such flow oscillations can form in shear layers (Yang et al., 2000) at the edges of spray streams, and in other areas in the flow due to pressure oscillations and induced oscillations as a result of flow-wall interaction. Although the assumptions of the model, described by Katoshevski et al (2005), might seem to be too simplistic for practical engineering applications, they allowed the authors to form a clear picture of the effect of the oscillating gas flow on droplet grouping. A more detailed analytical study of the equations introduced by Katoshevski et al (2005) was reported in the recent paper by Sazhin et al (2007). 
The physics behind the grouping phenomenon can be understood in terms of the fact that there is a non-zero relaxation time even for small particles (of several micro-meters in diameter) which has an important role in an oscillating motion of a group of particles/ droplets, or a rapidly changing particle local velocity field. When the flow velocity behaves as a propagating wave, droplets may experience acceleration or deceleration depending on their relative position in that wave in terms of their velocity. This brings to the separation between two adjacent particles/droplets in one location whereas in another location it brings particles/droplets closer together. Eventually, two stable areas in which droplets are being accumulated can be formed. Moreover, there is an interesting parallel between this grouping phenomenon (described by Katoshevski et al, 2005) and the phenomenon of trapping charged particles in plasma waves (Sazhin, 1993). This trapping phenomenon is expected in the case when the kinetic energy of charged particles in the frame of reference moving with the phase velocity of the wave is less than the potential energy of the wave in the same frame of reference. When trapping happens, the particles effectively start oscillating in the potential well of the wave in such a way that the particle average velocity becomes equal to the phase velocity of the wave. Note, however, that these oscillations are possible due to the fact that the dissipative forces in the case of plasma waves are generally relatively weak if plasma is rarefied (e.g. space plasma). In the case of droplet grouping in an oscillating flow, these dissipative forces are the dominant. The end result in both cases, however, is the same: droplets and particles move with velocities close to the phase velocities of the waves.

In this paper, the model described by Katoshevski et al (2005) is developed further. The above mentioned concept of trapping is applied to the analysis of droplet dynamics in an oscillating flow. The links between the concepts of grouping and trapping are investigated. The dynamics of mono-sized and two-sized droplets are considered, and the effects of 
evaporation on grouping behavior are studied. A two-way coupling between gas and droplet is also considered. The latter analysis is particularly important in dense sprays, where the kinetic energy of droplets is not negligible compared with the gas kinetic energy. Finally, a qualitative comparison is made between predictions of the model and in-house experimental data.

In the following section, the analysis of the equation of motion of droplets in an oscillating flow is presented. The analysis of this equation in the stationary frame of reference is supplemented by its analysis in the frame of reference moving with the wave phase velocity. A simplified analysis of droplet dynamics shows that they can be grouped in the vicinity of just one point per wavelength in this frame of reference. This is confirmed by the results of numerical analysis. The dynamics of the droplets' grouping coupled with evaporation is discussed in Section 3. In Section 4, a coupled solution for droplet and gas dynamics is presented to show the effect of droplets on the gas oscillatory motion and on grouping. In Section 5, the experimental set-up is described, and some predictions of the model are qualitatively compared with experimental data. The main results of the paper are summarised in Section 6.

\section{Droplet grouping and trapping}

Consider droplet motion in a one-dimensional periodic gas flow, described by the following equation (Katoshevski et al., 2005):

$v_{g}(t, x)=V_{a}-V_{b} \sin (k x-\omega t)$,

where $v_{g}(t, x)$ is the dimensional gas flow velocity at a time $t$ at a location $x, V_{a}$ is the dimensional mean flow velocity, $V_{b}$ is the dimensional amplitude of the velocity oscillation, $k$ is the wave number and $\omega$ is the angular velocity. Without loss of generality we assume that $V_{a}>0$ and $V_{b}>0$. The flow, described by Equation (1) has a period $L=2 \pi / k$ along 
the $x$-axis and a time period $T=2 \pi / \omega$. The dimensional phase velocity of the wave is $V_{w}=\omega / k$.

Normalising the velocities by $V_{w}$, the distance by $k^{-1}$ and the time by $\omega^{-1}$ leads to the following equation of droplet motion in the Stokes limit (droplet Reynolds number $\mathrm{Re}_{d}$, based on droplet diameter, is less than 2):

$$
\frac{d u_{d}}{d \tau}=\frac{1}{S t}\left(u_{g}-u_{d}\right)
$$

where $S t=\frac{1}{18} \frac{\rho_{l} \omega D_{d}^{2}}{\mu}$ is the Stokes number, $\rho_{l}$ is the liquid density, $D_{d}$ is the droplet diameter, and $\mu$ is the dynamic viscosity of the host gas; $u_{g}$ is the dimensionless gas velocity, $u_{d}$ is the dimensionless droplet velocity in the stationary frame of reference and $\tau$ is the dimensionless time.

The same Equation (2) will be used for droplet Reynolds numbers greater than 2 by assuming that: $S t=\frac{4 \rho_{l} \omega D_{d}}{3 C_{D} \rho_{g}\left|v_{g}-v_{d}\right|}$, where $v_{d}$ is the dimensional droplet velocity in the stationary frame of reference, the drag coefficient $C_{D}$ is estimated as (Seinfeld and Pandis, 1997): $C_{D}=\frac{24}{\operatorname{Re}_{d}}\left[1+0.15 \operatorname{Re}_{d}^{0.687}\right]$, when $2<\operatorname{Re}_{d}<500$, and $C_{D}=0.44$ when $\operatorname{Re}_{d} \geq 500$. At $\mathrm{Re}_{d} \leq 2$, the drag coefficient is estimated based on the well known formula $C_{D}=\frac{24}{\operatorname{Re}_{d}}$, which leads to the earlier presented expression for St. 
If we use the frame of reference moving with the wave phase velocity $\left(V_{w}=\omega / k\right)$ then the normalised wave velocity becomes 1 , and the non-dimensional droplet location becomes $X=k x-\omega t$. Hence Eq. (2) can be rearranged to:

$\frac{d U_{d}}{d \tau}=\frac{1}{S t}\left(U_{a}-1-U_{d}-U_{b} \sin X\right)$,

where the dimensionless droplet velocity $U_{d}$ is taken in the new frame of reference, the dimensionless values of $U_{a}$ and $U_{b}$ are in the stationary frame of reference. Note that all velocities are normalised by $V_{w}$.

It is easy to see that Equation (3) describes several types of solutions.

1) If $U_{a}-1-U_{d}>U_{b}$ then the condition $\frac{d U_{d}}{d \tau}>0$ is always satisfied and the droplet is expected to accelerate for all times and locations.

2) If $U_{a}-1-U_{d}<-U_{b}$ then the condition $\frac{d U_{d}}{d \tau}<0$ is always satisfied and the droplet is expected to decelerate for all times and locations.

3) If $U_{a}-1-U_{d}=-U_{b}$ or $U_{a}-1-U_{d}=U_{b}$ then $\frac{d U_{d}}{d \tau}<0$ or $\frac{d U_{d}}{d \tau}>0$ except when $X=-\pi / 2+2 \pi n$ or $X=\pi / 2+2 \pi n, n$ is an integer (when $\frac{d U_{d}}{d \tau}=0$ ), and the droplet is expected to decelerate or accelerate for most of the times and locations. The underlying physics of the processes in this case is similar to Cases 1 and 2 .

4) If $-U_{b}<U_{a}-1-U_{d}<U_{b}$ 
then $\quad \frac{d U_{d}}{d \tau}>0 \quad$ when $\quad U_{b}>U_{a}-1-U_{d}>U_{b} \sin X \quad$ and $\quad \frac{d U_{d}}{d \tau}<0 \quad$ when $-U_{b}<U_{a}-1-U_{d}<U_{b} \sin X$. This creates the necessary conditions when the droplets can become trapped in the vicinity of the points:

$X=X_{0}=\sin ^{-1}\left[\left(U_{a}-1-U_{d}\left(X_{0}, \tau\right)\right) / U_{b}\right]+2 \pi n$.

If $U_{d}$ in the right hand side of Equation (3) is so small that its contribution can be ignored then this equation can be solved analytically with the solution presented in terms of elliptical integrals (Abramovitz and Stegun, 1964). In the general case the solution of this equation needs to be performed numerically and this will be the subject of most of this paper.

In the limiting case when $U_{d}\left(X_{0}, \tau\right)=0$ Equation (4) is simplified to the following condition:

$\sin X_{0}=\beta \equiv\left(\left(U_{a}-1\right) / U_{b}\right)$.

In this case the acceleration of droplets also becomes zero. If Equation (5) has a solution (the absolute value of the right hand side is not greater than 1), then we would expect that these locations can potentially be reached by a number of droplets with various initial velocities and initial locations (in a certain range). This means that the droplets can become grouped. Hence, the necessary condition for grouping can be presented as $|\beta| \leq 1$. This condition is identical to the one obtained earlier by Katoshevski et al (2005) using a different approach.

Now we consider in some detail how the point $X_{0}$ can be reached by moving droplets. This can be viewed as a linear stability analysis, for a position close to $X_{0}$. Assuming that $X$ is close to $X_{0}$ we can present $X$ as:

$$
X=X_{0}+X_{1}
$$


where $X_{0}=\sin ^{-1}\left[\left(U_{a}-1\right) / U_{b}\right]+2 \pi n, \quad X_{1}<<1 . \quad$ In this case, $\sin X=\sin X_{0}+X_{1} \cos X_{0}$, where

$\cos X_{0}= \pm \sqrt{1-\left(\frac{U_{a}-1}{U_{b}}\right)^{2}}$

Hence, Equation (3) can be simplified to:

$\frac{d^{2} X_{1}}{d \tau^{2}}+\frac{1}{S t} \frac{d X_{1}}{d \tau}+\frac{U_{b} \cos X_{0}}{S t} X_{1}=0$

In the Stokes limit, St does not depend on droplet velocities. Hence, Equation (6) is a linear one, and its solution can be presented as:

$X_{1}=a_{1} \exp \left(\alpha_{1} \tau\right)+a_{2} \exp \left(\alpha_{2} \tau\right)$

where

$\alpha_{1,2}=\frac{1}{2 S t}\left[-1 \pm \sqrt{1-4 U_{b} S t \cos X_{0}}\right]$

coefficients $a_{1}$ and $a_{2}$ are determined from the initial conditions for $X_{1}$ and its first derivative.

The properties of Solution (7) depend on the values of $4 U_{b} S t \cos X_{0}$. If $0<4 U_{b} S t \cos X_{0} \leq 1$ then for any initial condition, $X_{1} \rightarrow 0$ without oscillations when $\tau \rightarrow \infty$. If $4 U_{b}$ St $\cos X_{0}>1$ then for any non-zero initial condition $X_{1} \rightarrow 0$ with oscillations at frequency $|\omega|=\frac{1}{2 S t}\left[\sqrt{4 U_{b} S t \cos X_{0}-1}\right]$ when $\tau \rightarrow \infty$. If $4 U_{b} S t \cos X_{0}<0$ then the solution predicts the possibility that $X_{1} \rightarrow \infty$ without oscillations when $\tau \rightarrow \infty$. This condition is expected when $\cos X_{0}<0$, but within the range of validity of our previous assumption of small $X_{1}$. 
Equation (5) has an infinite number of solutions for $X_{0}\left(X_{0}+2 \pi n\right)$ corresponding to various integer values $n$. For any given $n$, this equation has two solutions if $|\beta|$ is less than 1 ,. One of these solutions corresponds to $\cos X_{0}>0$, while the second solution corresponds to $\cos X_{0}<0$. Based on our previous analysis we can expect that only the first solution always describes the actual grouping (attractive trajectories). The second solution can describe the repelling trajectories which are not expected to lead to grouping. The attractive and repelling solutions of Equation (6) are schematically illustrated in Fig. 2. The existence of attractive and repelling particle trajectories was earlier reported by Katoshevski et al (2005) using a different approach.

The analysis presented above has obvious limitations, as it is restricted to the parts of droplet trajectories in the vicinity of the points $X_{0}$. The general analysis needs to be based on the numerical solution of Equation (2) in the stationary frame of reference, or Equation (3) in the frame of reference moving with the wave phase velocity. Firstly, this analysis was performed for n-dodecane $\left(\rho_{l}=600 \mathrm{~kg} / \mathrm{m}^{3}\right)$ droplets with diameters $D_{d}=20 \mu \mathrm{m}$ injected in gas with $V_{a}=60 \mathrm{~m} / \mathrm{s}$ and $V_{b}=20 \mathrm{~m} / \mathrm{s}$. The values of air dynamic viscosity and density are taken to be equal to $\mu=3.3 \times 10^{-5} \mathrm{Ns} / \mathrm{m}^{2}$ and $\rho_{g}=15.7 \mathrm{~kg} / \mathrm{m}^{3}$ respectively. These parameters are taken for gas temperature equal to $667 \mathrm{~K}$ and pressure equal to $3 \mathrm{MPa}$; the estimate of the temperature is discussed later in Section 3. These are realistic parameter values for the case of Diesel engine sprays (Crua, 2002). At the first stage, the effects of evaporation of these droplets are ignored. They will be taken into account later in Section 3. The wave angular velocity $\omega$ and wave number $k$ are taken to be equal to $10^{5} \mathrm{rad} / \mathrm{s}$ and $2000 \mathrm{rad} / \mathrm{m}$ respectively. This gives a wave phase velocity $V_{w}$ equal to $50 \mathrm{~m} / \mathrm{s} .21$ droplets were injected simultaneously over the range of $x$ from 0 to $3.14 \times 10^{-3} \mathrm{~m}$ (one wavelength) in such a way 
that the initial distances between them were equal to $1 / 20$ of the wavelength. The droplet velocities were taken to be equal to the velocity of gas at $t=0$. n-dodecane is widely used as a reasonable approximation to a realistic Diesel fuel (Sazhina et al, 2000). The Diesel fuel was chosen to allow us the qualitative comparison of the predictions of the model with "in-house" experimental data. Using these parameters we have $\beta=0.5$. Hence, the droplets can be trapped in the flow wave, and this can lead potentially to their grouping.

The plots of $x$ versus $t$ for 6 out of the 21 droplets in the stationary frame of reference are shown in Fig. 3. The numerical solution of Equation (2) was performed using the fourth order Runge-Kutte method. The time stepping was adaptive, starting with a very small time step and advancing to no more that one tenth of the time interval of the integration. The same method was used for obtaining the results shown in the following figures until Figure 14. As can be seen from Fig. 3, after a relatively short time (about $0.3 \mathrm{~ms}$ ) all droplets form two clear groups separated by the distance equal to the wavelength $3.14 \times 10^{-3} \mathrm{~m}$. The velocities of all droplets become equal to the wave phase velocity $(50 \mathrm{~m} / \mathrm{s})$. It is easy to show that this velocity is equal to the flow velocity at the location of the droplets.

As mentioned above, the initial droplet velocity is taken equal to the local gas velocity. This assumption can be supported by our calculations which show that if the initial droplet velocity is only $20 \%$ of the gas local velocity this will not have a noticeable effect on the curves shown in Fig. 3.

The plots of $X$ versus $V_{d}$, for the same values of parameters as in Fig. 3, but in the frame of reference moving with the wave are shown in Fig. 4. $V_{d}$ is the dimensional droplet velocity in the moving frame of reference. Droplet locations in this frame of reference, $X$, are presented in a dimensional form. As can be seen from this figure, all droplet trajectories converge on just two points, both of which correspond to $V_{d}=0$. The values of $X$ for these points are 
equal to $0.26 \mathrm{~mm}$ and $3.40 \mathrm{~mm}$ respectively. These points correspond to droplet grouping as shown in Fig. 3 for the stationary frame of reference. It can be seen that the general solution of Equation (5) for $\beta=0.5$ and $\cos X_{0}>0$, gives: $X_{0}=0.52+6.28 n$, where $n=0,1,2, \ldots$. For $n=0$ and $n=1$ this gives the same values of $X$, which were obtained from the numerical analysis. The solution of Equation (5), for which $\cos X_{0}<0$, describes the diverging trajectories which do not lead to droplet grouping.

The plots of $x$ versus $t$ in the stationary frame of reference for the same values of parameters as in Fig. 3, except for $V_{a}=70 \mathrm{~m} / \mathrm{s}$ and $V_{b}=15 \mathrm{~m} / \mathrm{s}$, are shown in Fig. 5. For these parameters we have $\beta=1.33$. Hence, the droplets are not expected to be trapped or grouped in the flow wave. This is confirmed for the trajectories shown in Fig 5. The droplets remain more or less evenly distributed in the space close to the wavelength. In fact this space increases slightly with time.

The plots of distance versus $V_{d}$ for the same values of parameters as in Fig. 5, but in the frame of reference moving with the wave phase velocity $\left(X, V_{d}\right)$ are shown in Fig. 6 . As can be seen from this figure, after the initial transient period, the velocities of all droplets begin to oscillate around the velocity close to the average flow velocity in the frame of reference of the wave $(20 \mathrm{~m} / \mathrm{s})$. The actual positions of droplets increase, and no grouping takes place. This is consistent with the prediction of Fig. 5.

At the next stage the effects of grouping of multi-sized droplets is investigated, based on the numerical solution of Equation (2) in the stationary frame of reference. This analysis has been performed for droplets with diameters $D_{d}=20 \mu \mathrm{m}$ and $D_{d}=40 \mu \mathrm{m}$ injected into gas 
with $V_{a}=60 \mathrm{~m} / \mathrm{s}$ and $V_{b}=20 \mathrm{~m} / \mathrm{s} . \omega$ and $k$ are taken to be equal to $10^{5} \mathrm{rad} / \mathrm{s}$ and 2000 $\mathrm{rad} / \mathrm{m}$. This gives a wave phase velocity $V_{w}$ equal to $50 \mathrm{~m} / \mathrm{s}$, as in Fig. 3 . Thirty droplets (15 of each size) have been injected simultaneously over the range of $x$ from 0 to $3.14 \times 10^{-3} \mathrm{~m}$ (one wavelength) in such a way that the initial distances between them are equal, and droplet sizes alternate. The droplet velocities are taken to be equal to the velocity of gas at $t=0$. The results for 10 trajectories are shown in Fig. 7. As can be seen from this figure, the small droplets become grouped after about $0.3 \mathrm{~ms}$. This reproduces the result shown in Fig. 3 . At the same time, the large droplets are not grouped although the condition $|\beta|<1$ is satisfied for both droplet sizes. Hence, the condition $|\beta|<1$ is necessary but not sufficient for grouping. This result is consistent with the one reported earlier by Katoshevski et al (2005). Note that the interactions between droplets are not taken into account in our analysis..

The trajectories of small droplets, shown in Fig. 7, in the moving frame of reference have already been presented in Fig. 4, and the grouping properties of these droplets were discussed. Similar trajectories for the larger droplets in the moving frame of reference are shown in Fig. 8. As follows from the latter figure, some of the large droplets are grouped (their trajectories are similar to those shown in Fig. 4) around $X=0.26 \mathrm{~mm}$, while most of them are not grouped (their trajectories are similar to those shown in Fig. 6). We cannot explain these properties of large droplet grouping based on the simplified analytical approach presented earlier. Note that for most of the trajectories of both large and small droplets, the values of $\operatorname{Re}_{d}$ are well above 2, and the Stokesian approximation is not valid. For larger droplets $\operatorname{Re}_{d}$ reached about 650, while for smaller droplets it reached about 250. The Stokesian approximation becomes valid only in the vicinity of the point $X_{0}$ in the moving frame of reference. 


\section{Grouping and evaporation}

Effects of droplet clustering on evaporation have been discussed in numerous papers including those by Dwyer et al (2000) and Harstad and Bellan (2001), and the results have been summarized in several monographs (e.g. Sirignano, 1999). The basic conclusion of all these studies is that clustering reduces the evaporation rate. The rate of this reduction, however, depends on specific droplet configuration, droplet and gas velocity and temperatures, and other factors. It is difficult to take into account all these factors in the simple model described in this paper.

In the model used in this paper, it is assumed that the main effect of grouping on evaporation comes not from the physical configuration of droplet positions, but from their Reynolds numbers. Moreover, the simplest possible version of the evaporation model is used, when the effects of heat-up and thermal radiation are ignored. In this case, the change of droplet diameter with time can be described by a particularly simple formula (Sirignano, 1999; Sazhin, 2006):

$D_{d}=\sqrt{D_{d 0}^{2}-\frac{8 k_{g} \Delta T}{L \rho_{l}}\left(1+0.3 \operatorname{Re}_{d}^{1 / 2} \operatorname{Pr}^{1 / 3}\right) t}$,

where $\Delta T=T_{g}-T_{d}, T_{g}$ is the gas temperature, $T_{d}$ is the droplet temperature (effects of temperature gradients inside droplets are ignored), $L$ is the specific heat of evaporation, $k_{g}$ is gas thermal conductivity, $\rho_{l}$ is the liquid density, $D_{d 0}$ is the droplet initial diameter, $\operatorname{Pr}$ is the Prandtl number, and the droplet Reynolds number is based of droplet diameter.

The more general model for droplet evaporation taking into account, the heat-up processes, effects of thermal radiation and temperature gradient inside droplets, and effects of variable 
transport coefficients was studied by Bertoli and na Migliaccio (1999), Sazhin et al (2005a,b, 2006) and Abramzon and Sazhin $(2005,2006)$. The analysis of these effects is beyond the scope of this paper.

As follows from the rigorous analysis of n-dodecane droplet evaporation in a hot gas, in conditions close to those in diesel engines ( $T_{g}$ close to $900 \mathrm{~K}$, pressure equal to $3 \mathrm{MPa}$ ), $T_{d}$ reaches the saturation level at temperatures close to $550 \mathrm{~K}$ (Sazhin et al, 2005b). Hence we can assume that $\Delta T=350 \mathrm{~K}$. In this case the physical properties of the ambient gas can be taken at temperature $T_{a v}=\left(T_{g}+2 T_{d}\right) / 3 \approx 667 \mathrm{~K}$. At this temperature the air thermal conductivity is taken to be equal to $0.05 \mathrm{~W} /(\mathrm{mK})$ and $\mathrm{Pr}=0.69$ (Incropera and DeWitt, 2002). Following Maxwell (1950) we can take $L=2.57 \times 10^{5} \mathrm{~J} / \mathrm{kg}, \rho_{l}=600 \mathrm{~kg} / \mathrm{m}$. This allows us to simplify the formula for $D_{d}$ to:

$$
D_{d}=\sqrt{D_{d 0}^{2}-0.91 \times 10^{-6}\left(1+0.265 \mathrm{Re}_{d}^{1 / 2}\right) t} .
$$

As follows from Equation (10), the rate of evaporation depends on $\mathrm{Re}_{d}$. Since the time evolutions of $\mathrm{Re}_{d}$ are expected to be different for droplets injected at different phases of the wave, we obtain different time evolutions of $D_{d}$. Here lies one of the effects of grouping on evaporation. The decreasing values of droplet diameters, in their turn, would lead to decreasing values of both $\operatorname{Re}_{d}$ and the Stokes number. Here lies an effect of evaporation on grouping. These two effects are obviously closely linked. Note that for the numerical analysis of Equation (10), the values of $\operatorname{Re}_{d}$ were assumed constant during the time step of integration $\Delta t$. They are allowed to change with time beyond this time step 
In addition to decreasing droplet diameters, the effect of evaporation leads to a reduction in the drag coefficient described by the following equation (Abramzon and Sirignano, 1989):

$C_{D}=\frac{C_{D 0}}{\left(1+B_{M}\right)^{\alpha}}$

where

$\alpha=1$ when $B_{M}<0.78$, and $\alpha=0.75$ when $B_{M} \geq 0.78, B_{M}$ is the Spalding mass transfer number, $C_{D 0}$ is the drag coefficient in the absence of evaporation. Equation (11) leads to the following modification of the expression for the Stokes number: $S t=S t_{0}\left(1+B_{M}\right)^{\alpha}$, where $S t_{0}$ is the Stokes number in the absence of evaporation.

The Spalding mass transfer number is defined as (Sirignano, 1999; Sazhin, 2006):

$B_{M}=\frac{Y_{f s}-Y_{f \infty}}{Y_{f s}-1}$

where $Y_{f s}$ and $Y_{f \infty}$ are mass fractions of fuel vapour near the surfaces of the droplets and in the ambient gas. We assume that $Y_{f \infty}=0$, and $Y_{f s}$ is estimated based on the following equation (Sazhin, 2006):

$Y_{f s}=\left[1+\left(\frac{p}{p_{f s}}-1\right) \frac{M_{a}}{M_{f}}\right]^{-1}$,

where $p$ is the ambient pressure, $p_{f s}$ is the fuel vapour partial pressure near the surface of the droplet, $M_{a}$ and $M_{f}$ are molar masses of air and fuel vapour. For n-dodecane: $M_{f}=170$ $\mathrm{kg} / \mathrm{kmol}$. 
Assuming that $n$-dodecane vapour partial pressure near the surface of the droplet is equal to the saturated pressure, and using data presented by Maxwell (1950), we obtained the following relation between $p_{f s}$ and the droplet surface temperature $T_{s}$ for $T_{s}>440 \mathrm{~K}$ :

$p_{f s}=p_{\text {ref }} \exp \left[\frac{T_{s}-T_{\text {ref }}}{a_{\text {pres }}}\right]$,

where $p_{\text {ref }}=46204.48 \mathrm{~Pa}, T_{\text {ref }}=449.87 \mathrm{~K}, a_{\text {pres }}=56.97 \mathrm{~K}$. Taking the same droplet temperature and ambient pressure as before $(550 \mathrm{~K}$ and $3 \mathrm{Mpa})$, and using the above relations, we obtain: $p_{f s}=0.268 \mathrm{MPa}, Y_{f s}=0.365$, and $B_{M}=0.575$. This allows us to write Expression (11) in a more specific form: $C_{D}=0.63 C_{D 0}$. Since the droplet temperature is assumed to be fixed for all droplets in the model under consideration, this correction is the same for all droplets.

To illustrate the effect of evaporation on grouping, droplets with diameters $D_{d}=60 \mu \mathrm{m}$ were injected into gas with $V_{a}=55 \mathrm{~m} / \mathrm{s}$ and $V_{b}=20 \mathrm{~m} / \mathrm{s}$. The wave angular velocity $\omega$ and wave number $k$ are taken to be equal to $10^{5} \mathrm{rad} / \mathrm{s}$ and $2000 \mathrm{rad} / \mathrm{m}$, as in the previous analysis. This gives a wave phase velocity $V_{w}$ equal to $50 \mathrm{~m} / \mathrm{s}$. 21 droplets were injected simultaneously as in the cases shown in Figs 3 and 5. The analysis is restricted to the time interval from 0 to 0.7 ms. During this time, none of the droplets entirely evaporated.

At first the effects of evaporation are ignored. The plots of $x$ versus $t$ for all droplets in the stationary frame of reference are shown in Fig. 9. This figure shows that no effects of grouping are observable (cf. the case shown in Fig. 5). The same plots as in Fig. 9, but with the effects of evaporation taken into account are shown in Fig. 10. As can be seen from this 
figure, the evaporation leads to droplet grouping, although the pattern of grouping turns out to be more complicated than shown in Fig. 3.

The time evolution of the droplet diameter squared for the same droplets as shown in Fig. 10, is presented in Fig. 11. As follows from this figure, the dependence of $D_{d}^{2}$ versus $t$ deviates considerably from the linear one. Moreover, the time evolutions of various droplets vary considerably. To understand the underlying physics behind these properties, the plots of $D_{d}^{2}$ versus $t$ for three typical droplets are highlighted in Fig. 11. These are the droplets with maximal, minimal and average rates of evaporation, which correspond to the initial locations $0.6283 \mathrm{~mm}, 0.157 \mathrm{~mm}$ and $1.885 \mathrm{~mm}$ respectively. The time evolutions of the Reynolds numbers for these three droplets are shown in Fig. 12. As follows from this figure, the values of $R e$ for all three droplets are rather complicated functions of time. This is reflected in the complicated dependence of $D_{d}^{2}$ on $t$, as shown in Fig. 11. The values of $\operatorname{Re}_{d}$ for the droplet with the fastest rate of evaporation are the largest for most of the time, while the values of $\mathrm{Re}_{d}$ for the droplet with the slowest rate of evaporation are the smallest. The plots of distance versus $V_{d}$ for some of the trajectories presented in Fig. 10, but in the frame of reference moving with the wave $\left(X, V_{d}\right)$ are shown in Fig. 13. The grouping tendency of some trajectories shown in this figure is clearly recognised. For most trajectories, however, there seems to be insufficient time for the grouping pattern to develop due to the limited lifetime of evaporating droplets. These results seem to be in general agreement with those shown in Fig. 10: smaller droplets have a higher tendency to group.

\section{Coupled solutions}

In order to study the effect of coupling between the continuous and discrete phases on the grouping process, the commercial CFD code FLUENT (Version 6.1) was used. Trajectories 
of discrete phase particles were calculated by integrating the force acting on these particles, in the Lagrangian frame of reference. The trajectories were calculated by stepwise integration over discrete time steps, with the integration time step set by FLUENT to obtain a minimum error. Coupling took place via the momentum transfer between the continuous and discrete phases (droplets). This coupling was described in terms of the momentum sink or source terms in the continuous phase momentum equation. A gas velocity profile described by Equation (1) was imposed at the inlet.

The computational geometry was taken to be $2 \mathrm{D}$ and axis-symmetrical, as shown in Fig. 14 . We believe that this geometry can approximate reasonably well the $1 \mathrm{D}$ processes described so far in the paper. We could not use the $1 \mathrm{D}$ geometry directly due to software limitations. The dimensions of the domain are shown in this figure. 250 quadrilateral cells in the flow directions were used. The mesh density has been increased until grid independent solution was achieved. The flow and wave phase velocities are taken as follows: $V_{a}=0.7 \mathrm{~m} / \mathrm{s}$, $V_{b}=0.5 \mathrm{~m} / \mathrm{s}$ and $V_{\omega}=0.62 \mathrm{~m} / \mathrm{s}$. This choice of velocities was made for better illustration of the phenomenon, and is not directly linked with engine parameters. For these velocities $\beta=0.16$, and stable grouping is expected. Locations of the injected droplets are also shown in the same Fig. 14. The initial location of these droplets was one wave length from the entrance (based on numeric considerations), and they were evenly spread over the second wavelength. Droplets with $D_{d}=20 \mu \mathrm{m}$ were injected. 16 droplets were calculated.

Fig 15 shows the trajectories of droplets in the non-coupled case (a) and in the coupled one (b). Comparing Figs $15 \mathrm{a}$ and $15 \mathrm{~b}$, one can see that coupling between two phases tends to decrease the grouping. This can be related to the fact that the presence of droplets suppresses 
the flow oscillations and thus increases the local $\beta$ values. This is expected to lead to nongrouping tendency, which is confirmed by the observation of trajectories shown in Fig. 15b.

The results of calculations, using the same values of parameters as in Figs. 15ab, but obtained using the 1D model are shown in Fig. 15c. The comparison between Figs. 15a, 15b and $15 \mathrm{c}$ show that the processes described by the $1 \mathrm{D}$ model are in qualitative agreement with those described by FLUENT using the uncoupled 2D solution.

The next section will be focused on the qualitative comparison of the predictions of the model with available in-house experimental data referring to observations of sprays in Diesel engines.

\section{Experimental results}

The spray rig facility at the University of Brighton was designed specifically to enable the gaps in the current literature (spray and combustion analysis of injections into environments up to above $6 \mathrm{MPa}$ ) to be addressed, in addition to providing a means of testing sprays at conditions anticipated for the next generation of Diesel engines. This rapid compression machine is based around a single cylinder Ricardo Proteus test engine which was converted to two-stroke cycle operation by the addition of inlet and exhaust ports in the cylinder liner. The engine had a bore of $135 \mathrm{~mm}$, a stroke of $150 \mathrm{~mm}$, and a displacement of 2.2 litres. The compression ratio of the engine was reduced to $9: 1$ to further increase the volume available for the optical chamber. In-cylinder temperatures and pressures representative of a modern engine were obtained by increasing the boost pressure and temperature up to $0.8 \mathrm{MPa}$ and $100^{\circ} \mathrm{C}$ respectively. An optical chamber $80 \mathrm{~mm}$ in length and $50 \mathrm{~mm}$ in diameter was fitted onto the cylinder head to enable the full length of the developed fuel spray to be viewed. This chamber provided a near quiescent high-pressure environment, with realistic in-cylinder 
conditions being achieved by conditioning of the intake air. The fuel injector used was a modern electro-magnetically actuated common rail injector. For all experimental work lowsulphur Esso AF1313 Diesel reference fuel was used. Optical access into the chamber was provided by four removable sapphire windows $25 \mathrm{~mm}$ wide and $55 \mathrm{~mm}$ high. The digital video camera used in this series of experiments was a Phantom V7.1. The best compromise between acquisition rate and image resolution was obtained with a frame rate of $34300 \mathrm{fps}$, with a corresponding resolution of $128 \times 320$ pixels. The exposure time was independent of the frame rate, and set to 2 microseconds. The processing of the videos was performed by purpose-developed software that measured the spray penetration length and spray cone angle at each video frame after suitable pixel thresholding (Crua, 2002).

A qualitative comparison between the predictions of the model and experimental results performed using the above described set-up is presented in Fig. 16. On the right hand side of the figure a typical spray image is shown. From this image, a number of recognised groups can be clearly observed, the spacing between them being approximately equal to $3 \mathrm{~mm}$. This served as an input for the model. The model predictions are shown on the left hand side. The average droplet diameter was estimated as $10 \mu \mathrm{m}$ and the value of $\omega$ was estimated from the distance between groups $(3 \mathrm{~mm})$, and their velocity $V_{w}=50 \mathrm{~m} / \mathrm{s}$. The latter was estimated from the measurements, assuming the phase velocity lags behind the mean flow velocity, found to be $V_{a}=60 \mathrm{~m} / \mathrm{s}$. The angular velocity was determined to be $\omega=10^{5} \mathrm{rad} / \mathrm{s}$. There is much uncertainty regarding the value of $V_{b}$, which we took to be $6 \mathrm{~m} / \mathrm{s}$. In this case, $\beta=1.67$. Although in this case the model predicts non-grouping behaviour, some traces of weak grouping can still be seen. The overall grouping characteristics predicted by the model are rather similar to those observed experimentally. For larger $V_{b}$, much more pronounced grouping would have been predicted, but this would not be supported by experimental data. 


\begin{tabular}{|c|c|c|c|c|c|c|}
\hline Points & Frame & $X($ pix $)$ & $Y(p i x)$ & d (pix) & d $(\mathrm{mm})$ & $V(\mathrm{~m} / \mathrm{s})$ \\
\hline \multirow{2}{*}{1} & 97 & 74 & 91 & & & \\
\hline & 98 & 76 & 109 & 18.11 & 2.52 & 86 \\
\hline \multirow{2}{*}{2} & 97 & 68 & 98 & & & \\
\hline & 98 & 68 & 115 & 17.00 & 2.36 & 81 \\
\hline \multirow{2}{*}{3} & 97 & 76 & 116 & & & \\
\hline & 98 & 76 & 129 & 13.00 & 1.81 & 62 \\
\hline \multirow{2}{*}{4} & 97 & 61 & 251 & & & \\
\hline & 98 & 61 & 261 & 10.00 & 1.39 & 48 \\
\hline
\end{tabular}

Table 1. Locations and velocities of the points shown in Fig. 17

The main uncertainty in the interpretation of the results presented in Fig. 16 lies in the fact that we cannot determine the dynamics of fine structures of the spray based on a single image. To eliminate this uncertainty two consecutive images (frames), separated by $1 / 34300=$ $2.91545 \times 10^{-5}$ seconds in time, were compared. These are shown in Fig. 17 for frames 97 and 98. Locations of four identifiable structures are marked as points 1,23 and 4. Taking the $x$ axis as perpendicular to the axis of the spray and the $y$-axis as along the axis of the spray, the values of $x$ and $y$ for all four points in frame 97 were found. These are shown in Table 1 in pixels ( 1 pixel is approximately equal to $0.139 \mathrm{~mm}$ ). Then the values of $x$ and $y$ for all four points in frame 98 were found and presented in Table 1. The observation of the values of $x$ and $y$ for both frames shows that $x$ is practically unchanged from one frame to another, while the changes in $y$ are significant. This indicates that the fine structures in the spray propagate mainly along the $y$ axis and this is compatible with the main assumption of the 1D model developed in this paper. The distance between locations of the points in frames 97 and 98 are shown in Table 1 in pixels and mm. Remembering the abovementioned time separation between frames, the velocities of points 1-4 were calculated. The results are shown in Table 
1. It follows from this table that velocity decreases with distance, as expected (Sazhin et al, 2001, 2003). The velocity values were compared with the velocities of spray tips for other frames but for about the same values of $y$ as for points 1- 3 shown in Fig. 17. The difference between these velocities and the corresponding velocities calculated from Fig. 17 and shown in Table 1 was generally not more than about $10-15 \%$. This is compatible with the parameter values taken for calculations, the results of which were presented in the previous sections.

\section{Conclusions}

A new approach to the analysis of droplet grouping in an oscillating gas flow is suggested. This is based on the analysis of droplet trajectories in the frame of reference moving with the phase velocity of the wave. In the case of grouping, droplet trajectories in this frame of reference converge to the points for which $X_{0}=\sin ^{-1}\left[\left(U_{a}-1\right) / U_{b}\right]+2 \pi n$, provided that $\cos X_{0}>0$ ( $X_{0}$ is the distance in the moving frame of reference, normalised by the wave number; $U_{a}$ and $U_{b}$ are the flow translational velocity in the stationary frame of reference and the amplitude of flow oscillations, normalised by the wave phase velocity, respectively). In the case of non-grouping, droplet trajectories in this frame of reference oscillate around the translational velocity of the flow in the same frame of reference. In agreement with the previously reported results, it is shown that the necessary condition for grouping is $\left[\left|U_{a}-1\right| / U_{b}\right]<1$. In the case of two size droplets, the grouping pattern of larger droplets can become complicated; some are grouped, while others are not, although the condition 
$\left[\left|U_{a}-1\right| / U_{b}\right]<1$ was valid for all droplets. For smaller droplets much more stable grouping was observed. The effect of droplet evaporation is studied in the limiting case when the contribution of the heat-up period can be ignored. It is shown that evaporation can lead to droplet grouping even in the case when the non-evaporating droplets are not grouped. This is

related to the decrease of droplet diameters during the evaporation process. The predicted dependence of droplet diameter squared versus time is shown to deviate considerably from the linear function, due to the complicated and not monotonous dependence of droplet Reynolds number on time. The effect of the oscillating flow is shown to lead to noticeably different time evolutions of droplet diameter for different droplets although the initial droplet diameters were the same. Coupling between the momentum equations of the two phases shows that the presence of the droplets leads to damping of the local host gas velocity oscillations, and this in turn decreases grouping tendency.

The predicted grouping patterns of the droplets are shown to be in qualitative agreement with available in-house experimental observations of Diesel sprays. Analysis of the motion of the recognisable features of these sprays shows that these features move mainly along the spray axis. This is compatible with one of the main assumptions of the model, developed in the paper, that the directions of the gas and wave phase velocities are the same.

\section{Acknowledgment}

The authors are grateful to EPSRC (Grant EP/D002044/1), UK, for financial support and to the EPSRC Instrument Loan Pool for supplying the high-speed video camera used in this study. 


\section{References}

Abramovitz, M., Stegun, I.A., 1964. Handbook of Mathematical Functions, National Bureau of Standards, USA.

Abramzon, B., Sazhin, S., 2005. Droplet vaporization model in the presence of thermal radiation. Int J Heat Mass Transfer 48, 1868-1873.

Abramzon, B., Sazhin, S., 2006. Convective vaporization of fuel droplets with thermal radiation absorption. Fuel 85, 32-46.

Bertoli, C., na Migliaccio, M.A., 1999. Finite conductivity model for diesel spray evaporation computations. Int J Heat and Fluid Flow 20. 552-561.

Crua, C., 2002. Combustion Processes in a Diesel Engine. PhD Thesis, University of Brighton (UK) (www.crua.net/thesis).

Demoulin, F.X., Borghi, R., 2003. Modeling of turbulent spray combustion with application to diesel like experiment. Combustion and Flame 129, 281-293.

Dwyer, H.A., Stapf, P., Maly, R., 2000. Unsteady vaporization and ignition of a threedimensional droplet array. Combustion and Flame 121, 181-194.

Gao, Z., Mashayek, F., 2004. Stochastic modeling of evaporating droplets polydispersed in turbulent flows. Int J Heat and Mass Transfer 47, 4339-4348. 
Gouesbet, G., Berlemont, A, 1999. Eulerian and Lagrangean approaches for predicting the behaviour of discrete particles in turbulent flows. Prog Energy Combustion Science 25, 133159.

Harstad, K., Bellan, J., 2001. Evaluation of commonly used assumptions for isolated and cluster heptane drops in nitrogen at all pressures. Combustion and Flame 121, 1861-1879.

Incropera, F.P., DeWitt, D.P., 2002. Fundamentals of heat and mass transfer. New York: John Wiley \& Sons. Fifth edition.

Katoshevski, D., Dodin, Z., Ziskind, G., 2005. Aerosol clustering in oscillating flows: mathematical analysis, Atomization and Sprays 15, 401-412

Loth, E., 2000. Numerical approaches for motion of dispersed particles, droplets and bubbles. Prog Energy Combustion Science 26,161-223.

Mashayek, F., Pandya, R.V.R., 2003. Analytical description of particle laden flows. Prog Energy Combustion Science 29, 329-378.

Maxwell, J.B., 1950. Data book on hydrocarbons: application to process engineering. New York: D. van Nostrand Company.

Sazhin, S.S., 1993. Whistler-Mode Waves in a Hot Plasma. Cambridge: Cambridge University Press. 
Sazhin, S.S., 2006. Advanced models of fuel droplet heating and evaporation. Progress in Energy and Combustion Science 32, 162-214.

Sazhin, S.S., Feng, G. and Heikal, M.R., 2001. A model for fuel spray penetration. Fuel 80, $2171-2180$

Sazhin, S.S, Crua, C., Kennaird, D. and Heikal, M.R., 2003. The initial stage of fuel spray penetration. Fuel 82, 875-885.

Sazhin, S.S., Abdelghaffar, W.A., Krutitskii, P.A., Sazhina, E.M., Heikal, M.R., 2005a. New approaches to numerical modelling of droplet transient heating and evaporation. Int $\mathrm{J}$ Heat Mass Transfer 48, 4215-4228.

Sazhin, S.S., Abdelghaffar, W.A., Sazhina, E.M., Heikal, M.R., 2005b. Models for droplet transient heating: effects on droplet evaporation, ignition, and break-up, Int $\mathrm{J}$ Thermal Science 44, 610-622.

Sazhin, S.S., Kristyadi, T., Abdelghaffar, W.A., Heikal, M.R., 2006, Models for fuel droplet heating and evaporation: comparative analysis, Fuel 85, 1613-1630.

Sazhin S.S., Shakked, T., Sobolev, V., Katoshevski, D., 2007, Particle grouping in oscillating flows, European J of Mechanics B/Fluids (in press). 
Sazhina, E.M., Sazhin, S.S., Heikal, M.R., Babushok, V.I., Johns, R., 2000. A detailed modelling of the spray ignition process in Diesel engines, Combustion Science and Technology 160, 317-344.

Seinfeld, J.H., Pandis, S.N., 1997. Atmospheric Chemistry and Physics - From Air Pollution to Climate Change, John Wiley \& Sons.

Sirignano, W.A., 1999. Fluid dynamics and Transport of Droplets and Sprays, Cambridge University Press, Cambridge.

Yang X., Thomas N.H., and Guo L.J., 2000. Particle dispersion in organized vortex structures within turbulent free shear flows, Chem. Eng. Sci. 55, 1305-1324.

\section{Figure captions}

Figure 1. Schematic presentation of grouping of droplets in an oscillating flow field. The values of flow velocity are indicated by the sizes of the arrows. The droplets are introduced from the inlet. As they travel downstream grouping and evaporation take place.

Figure 2. Graphical solutions of Equation (5) for $0<\beta=\beta_{1}<1$ and $-1<\beta=\beta_{2}<0$. Filled circles show the stable solutions corresponding to $\cos X_{0}>0$, unfilled circles show the unstable solutions corresponding to $\cos X_{0}<0$. 
Figure 3. The plots of $x$ versus $t$ (dimensional) for 6 droplets in the stationary frame of reference. The following parameter values were used: $D_{d}=20 \mu \mathrm{m}, V_{a}=60 \mathrm{~m} / \mathrm{s}, V_{b}=20$ $\mathrm{m} / \mathrm{s}, \mu=3.3 \times 10^{-5} \mathrm{Ns} / \mathrm{m}^{2}, \rho_{g}=15.7 \mathrm{~kg} / \mathrm{m}^{3}$. Evaporation of droplets is ignored. The wave angular velocity $\omega$ and wave number $k$ are taken to be equal to $10^{5} \mathrm{rad} / \mathrm{s}$ and $2000 \mathrm{rad} / \mathrm{m}$ respectively. This gives a wave phase velocity $V_{w}$ equal to $50 \mathrm{~m} / \mathrm{s}$. The droplet velocities are taken to be equal to the velocity of gas at $t=0$. Using these parameters we have $\beta=0.5$. After a relatively short time (about $0.3 \mathrm{~ms}$ ) all droplets form two clear groups separated by the distance equal to the wavelength $3.14 \times 10^{-3} \mathrm{~m}$. The velocities of all droplets become equal to the wave phase velocity. This velocity is equal to the flow velocity at the location of the droplets.

Figure 4. The plots of $X$ versus $V_{d}$ (dimensional), for the same parameter values as in Fig. 3, but in the frame of reference moving with the wave. All droplet trajectories converge to two points, both of which correspond to $V_{d}=0$. The values of $X$ for these points are equal to 0.26 $\mathrm{mm}$ and $3.40 \mathrm{~mm}$ respectively. These points correspond to the solution of Equation (5) for which $\cos X_{0}>0$. The times elapsed after the injection are indicated as symbols on the curves.

Figure 5. The plots of $x$ versus $t$ in the stationary frame of reference for the same parameter values as in Fig. 3, except for $V_{a}=70 \mathrm{~m} / \mathrm{s}$ and $V_{b}=15 \mathrm{~m} / \mathrm{s}$. For these parameters we have $\beta=1.33$. The droplets remain more or less evenly distributed in the space close to the wavelength. In fact this space increases slightly with time. They are not trapped or grouped in agreement with the results of the simplified analysis. 
Figure 6. The plots of $X$ versus $V_{d}$ for the same parameter values as in Fig. 5, but in the frame of reference moving with the wave phase velocity. After the initial transient period, the velocities of all droplets begin to oscillate around the average flow velocity in the frame of reference of the wave $(20 \mathrm{~m} / \mathrm{s})$. The actual positions of droplets monotonously increase, and no grouping takes place. The times elapsed after the injection are indicated as symbols on the curves.

Figure 7. The plots of $x$ versus $t$ in the stationary frame of reference for two groups of droplets with diameters $D_{d}=20 \mu \mathrm{m}$ and $D_{d}=40 \mu \mathrm{m}$ (solid and dashed respectively) injected into gas with $V_{a}=60 \mathrm{~m} / \mathrm{s}$ and $V_{b}=20 \mathrm{~m} / \mathrm{s}$. The wave frequency $\omega$ and wave number $k$ are taken to be equal to $10^{5} \mathrm{rad} / \mathrm{s}$ and $2000 \mathrm{rad} / \mathrm{m}$. This gives a wave phase velocity $V_{w}$ equal to $50 \mathrm{~m} / \mathrm{s}$. The droplet velocities are taken to be equal to the velocity of gas at $t=0$. The small droplets become grouped after about $0.3 \mathrm{~ms}$. This reproduces the result shown in Fig. 3. The large droplets are not grouped although the condition $\beta<1$ is satisfied for both droplet sizes.

Figure 8. The trajectories of the same large droplets as shown in Fig. 7, but in the moving frame of reference. Some of these droplets are grouped (their trajectories are similar to those shown in Fig. 4), while most of them are not grouped (their trajectories are similar to those shown in Fig. 6).

Figure 9. The plots of $x$ versus $t$ for non-evaporating droplets in the stationary frame of reference. The following parameter values were used: $D_{d}=60 \mu \mathrm{m}, V_{a}=55 \mathrm{~m} / \mathrm{s}, V_{b}=20$ 
$\mathrm{m} / \mathrm{s}$. The wave angular velocity $\omega$ and wave number $k$ are taken to be equal to $10^{5} \mathrm{rad} / \mathrm{s}$ and $2000 \mathrm{rad} / \mathrm{m}$, as in the previous analysis. This gives a wave phase velocity $V_{w}$ equal to $50 \mathrm{~m} / \mathrm{s}$.

Figure 10. The same as Fig. 9, but for evaporating droplets. The results of the analysis are presented for the time interval from 0 to $0.6 \mathrm{~ms}$. During this time, none of the droplets is entirely evaporated.

Figure 11. Plots of $D_{d}^{2}$ versus time $t$ for the same droplets as shown in Fig. 10. The plots for three typical droplets are highlighted. These are the droplets with maximal (short dashes), minimal (long dashes) and average (solid) rates of evaporation.

Figure 12. The time evolutions of the Reynolds numbers for three droplets highlighted in Fig. 11.

Figure 13. The trajectories of the same evaporating droplets as shown in Fig. 10, but in the moving frame of reference. The times elapsed after the injection are indicated as symbols on the curves.

Figure 14. The geometry used for the coupled solution. The locations of the points of droplet injection are shown by dots on the axis of symmetry. The inlet is at the axis of symmetry. The symmetry of the problem allows us to consider only half of the domain.

Figure 15. Comparison between trajectories of non-evaporating droplets obtained from FLUENT 2D non-coupled (a) and coupled solutions (b) and the solution obtained using the 1D model (c). Non-dimensional distances and time are used in this figure ( $x$ and $\tau$ ). The flow 
and wave phase velocities are taken as follows: $V_{a}=0.7 \mathrm{~m} / \mathrm{s}, V_{b}=0.5 \mathrm{~m} / \mathrm{s}$ and $V_{\omega}=0.62$ $\mathrm{m} / \mathrm{s}$. Droplets with $D_{d}=20 \mu \mathrm{m}$ were injected.

Figure 16. A qualitative comparison between an experimentally observed diesel spray (right) and the predicted droplet trajectories (left). The spray was recorded $2.0 \mathrm{~ms}$ after the start of injection. The in-cylinder and injection pressures were 6 and $160 \mathrm{MPa}$, respectively. Air temperature and density were equal to $590 \mathrm{~K}$ and $36 \mathrm{~kg} / \mathrm{m}^{3}$. Grouping of droplets is observed at the shear flows at the edges of the spray. The model shows traces of grouping behavior (weak grouping) The horizontal dashed line marks the distance of $2.5 \mathrm{~cm}$. The horizontal solid lines indicate the location of the groups.

Figure 17. Images of fuel sprays separated by $1 / 34300=2.91545 \times 10^{-5}$ seconds . (frames 97 and 98). Locations of four identifiable structures are marked as points 1, 2, 3 and 4 on both images. $x$ and $y$ coordinates for these points in both frames are st pixels (1 pixel is approximately equal to $0.139 \mathrm{~mm}$ ). 


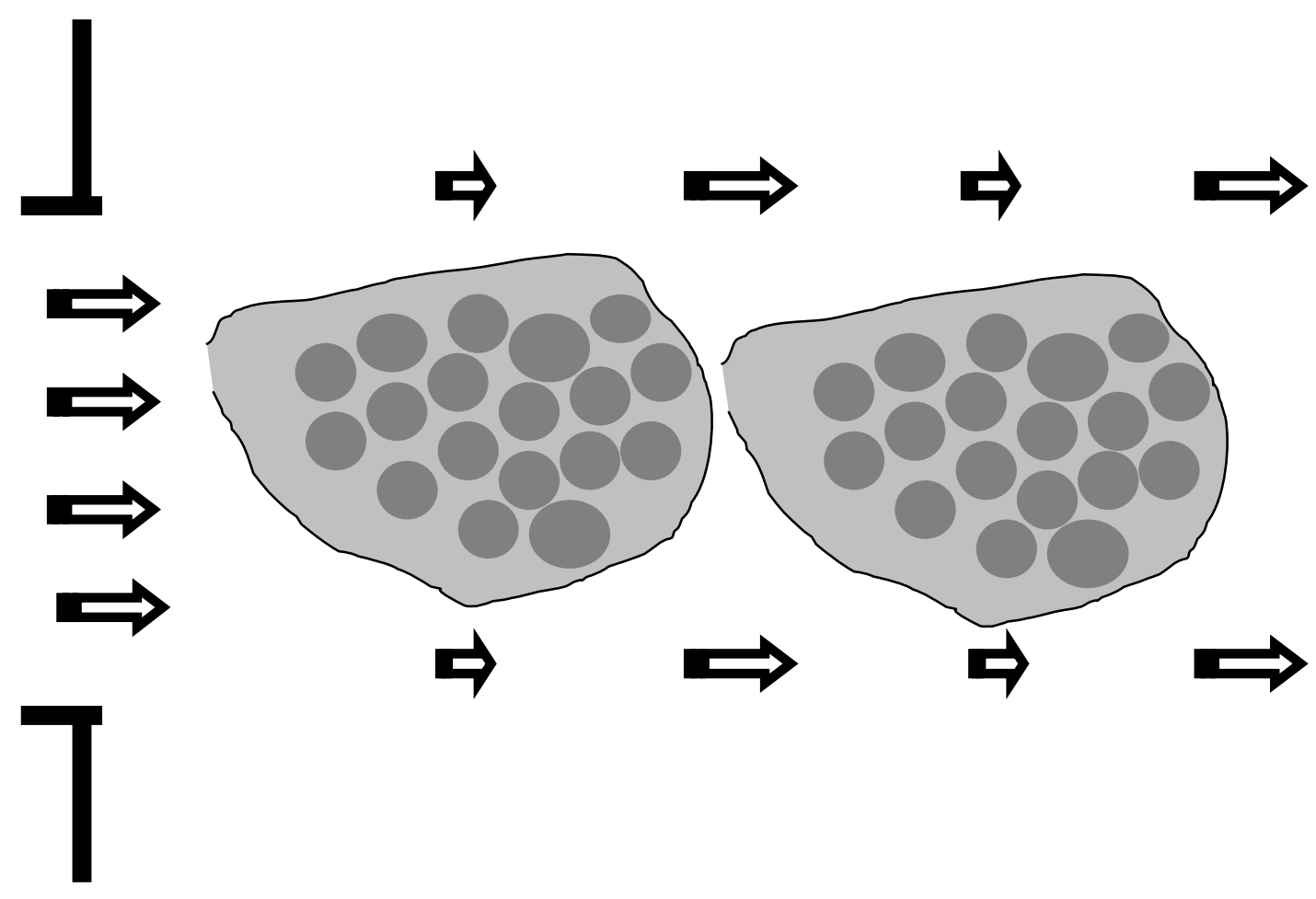

Fig.1 


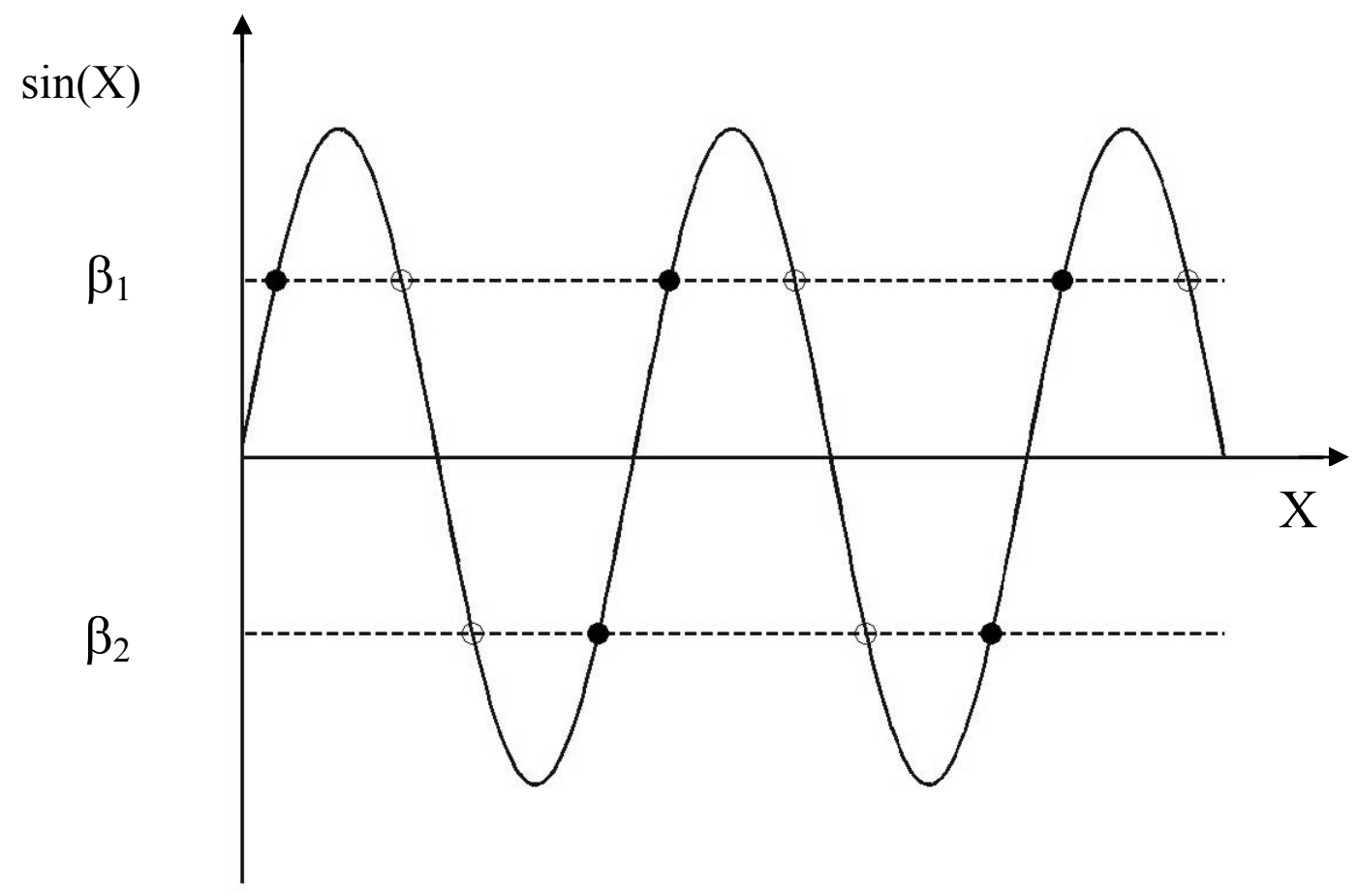

Fig. 2 


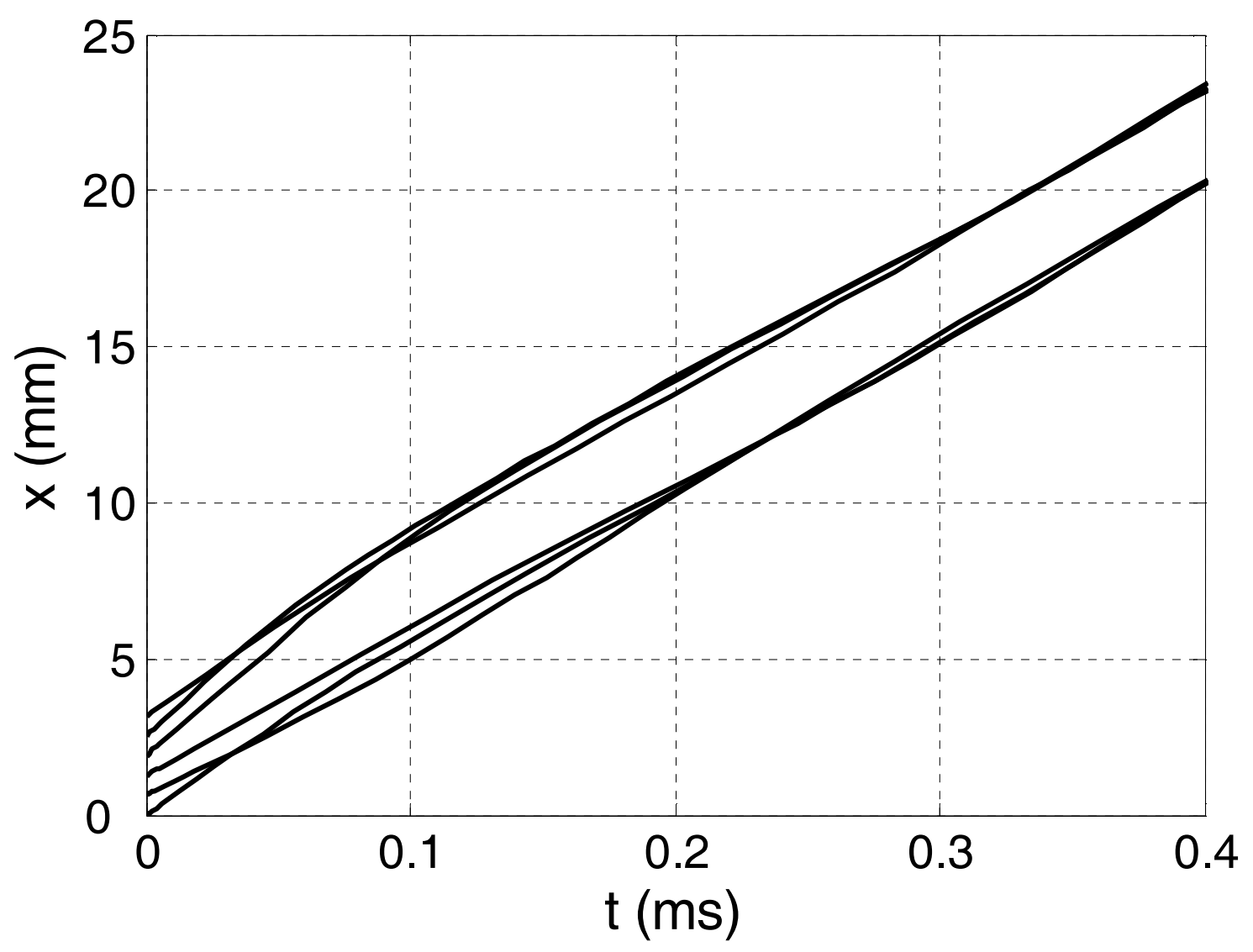

Fig. 3 


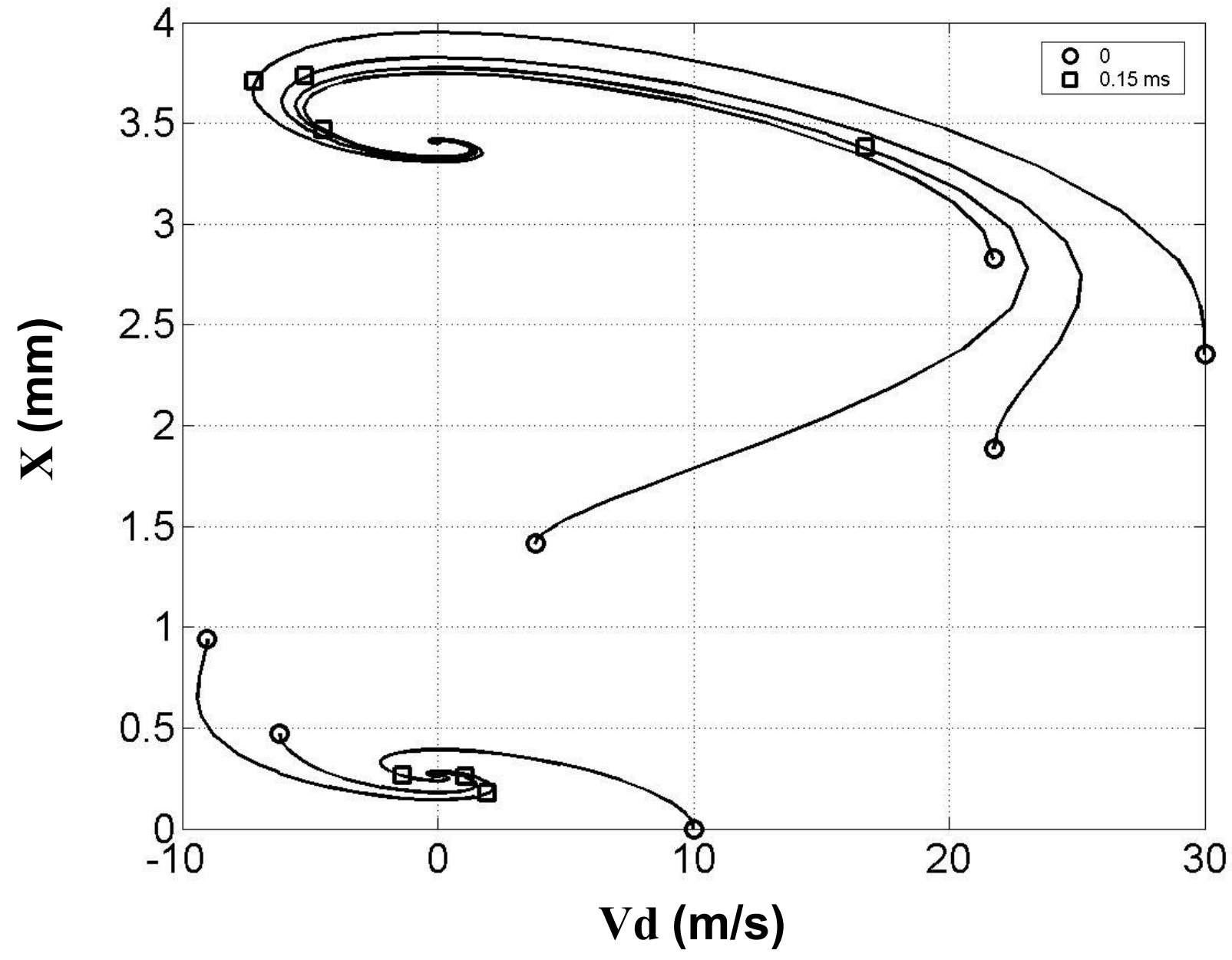

Fig. 4 


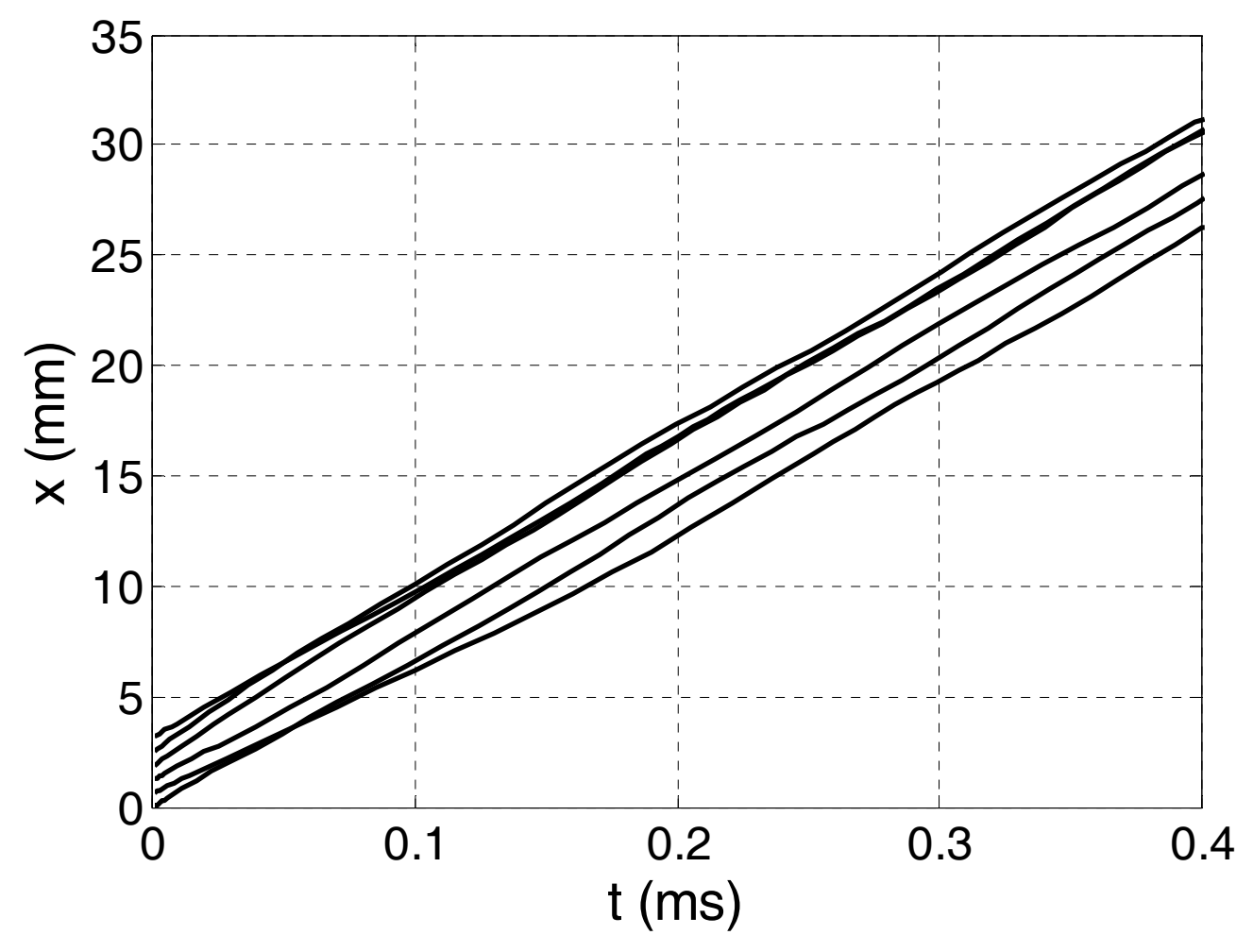

Fig. 5 


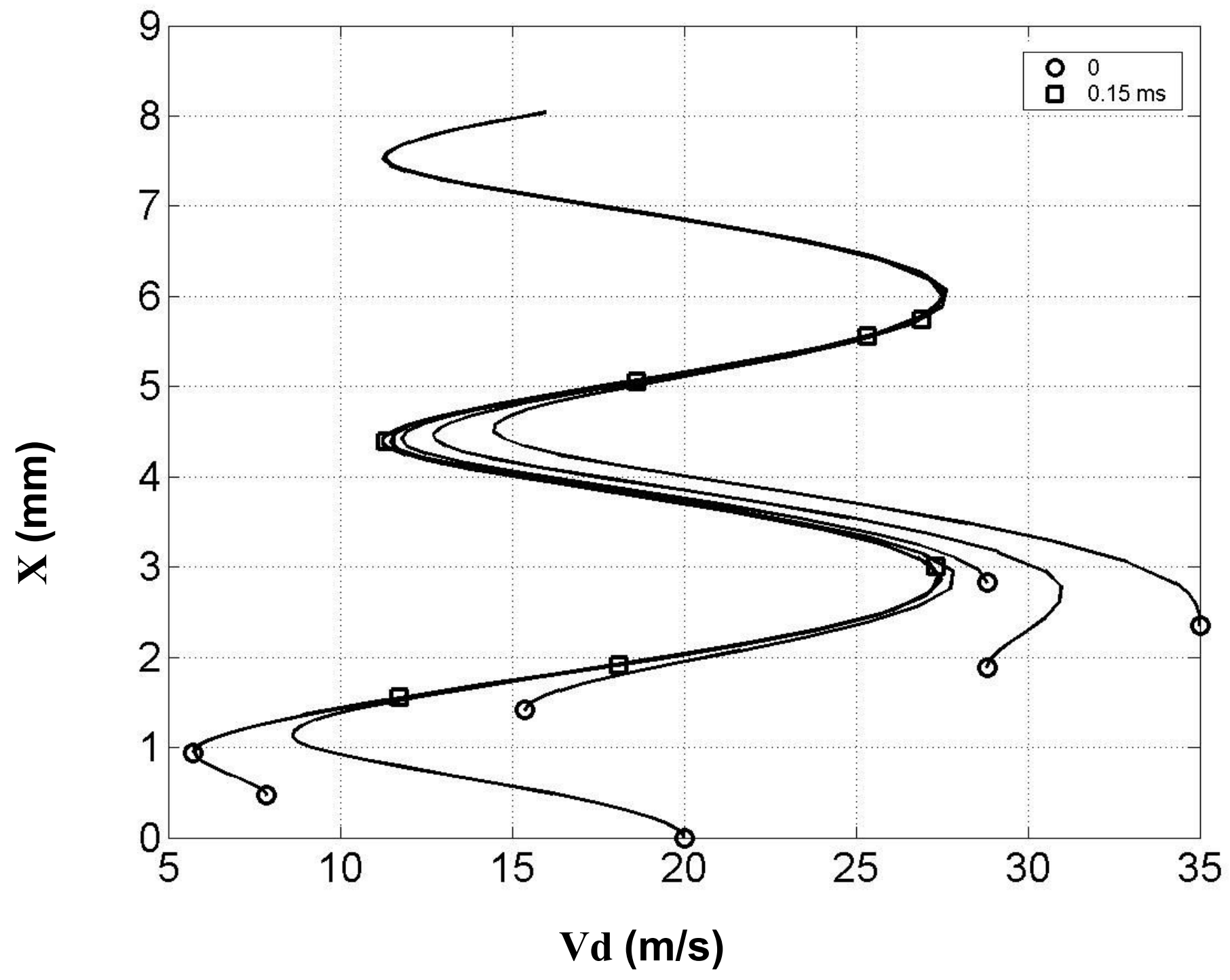

Fig. 6 


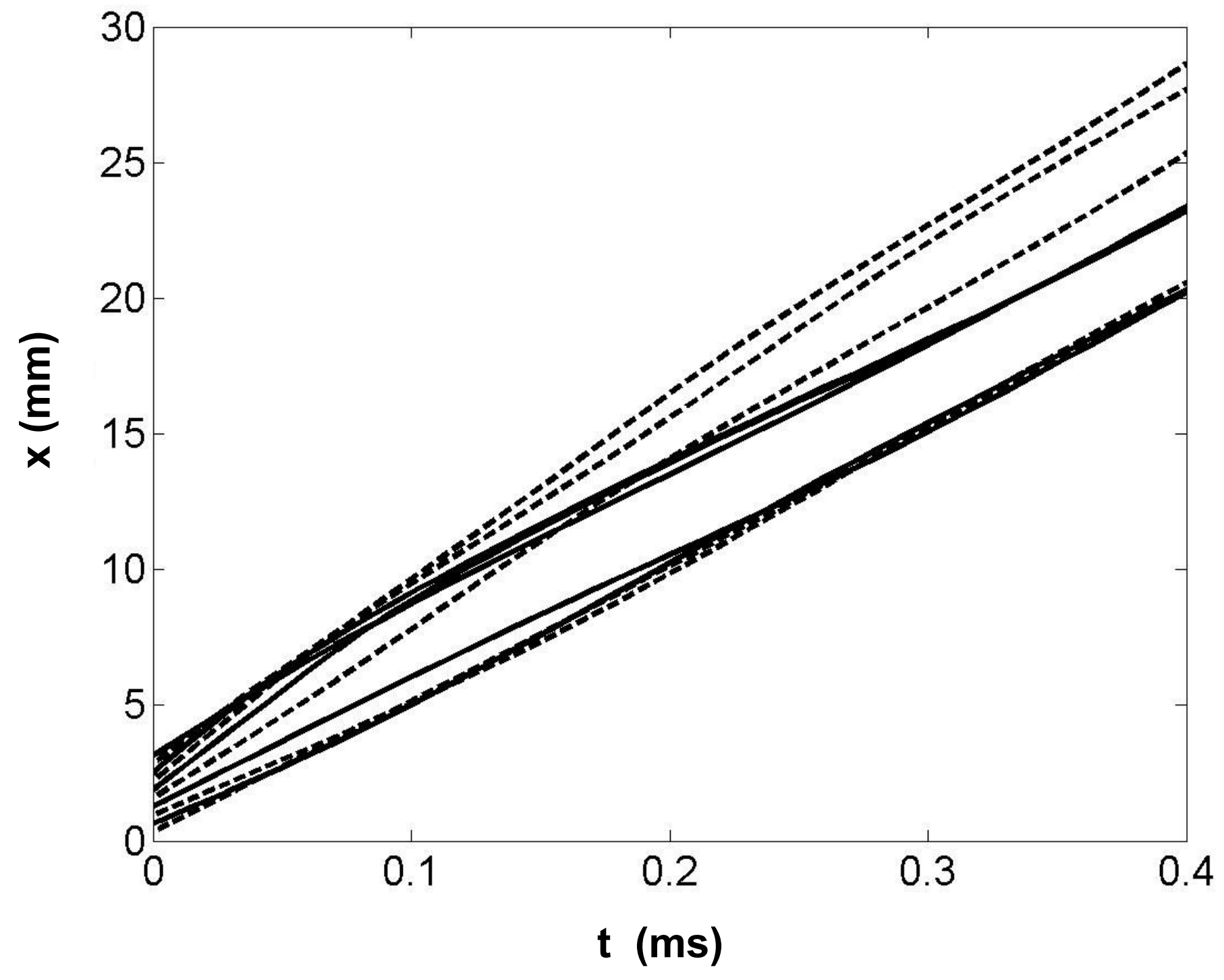

Fig. 7 


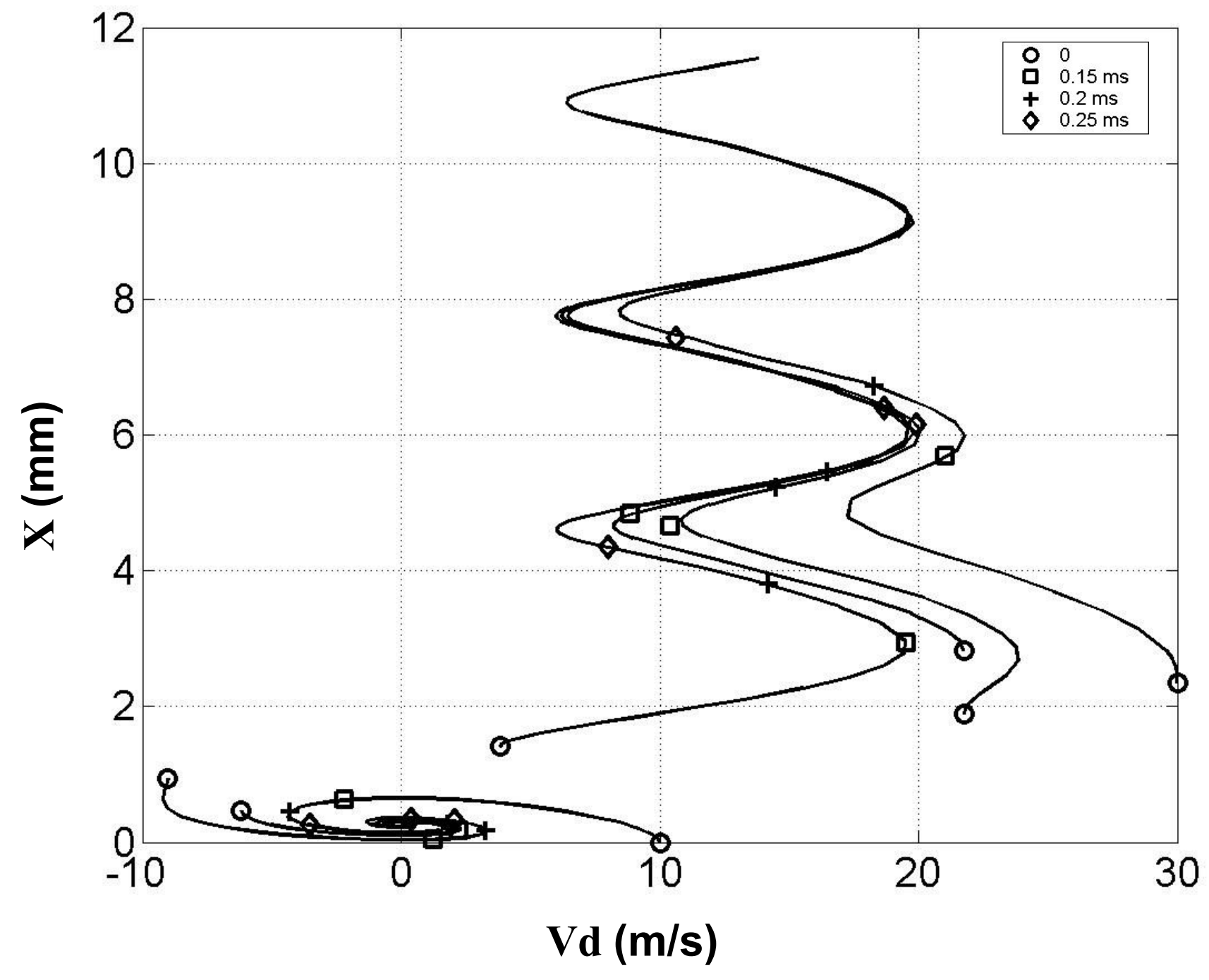

Fig. 8 


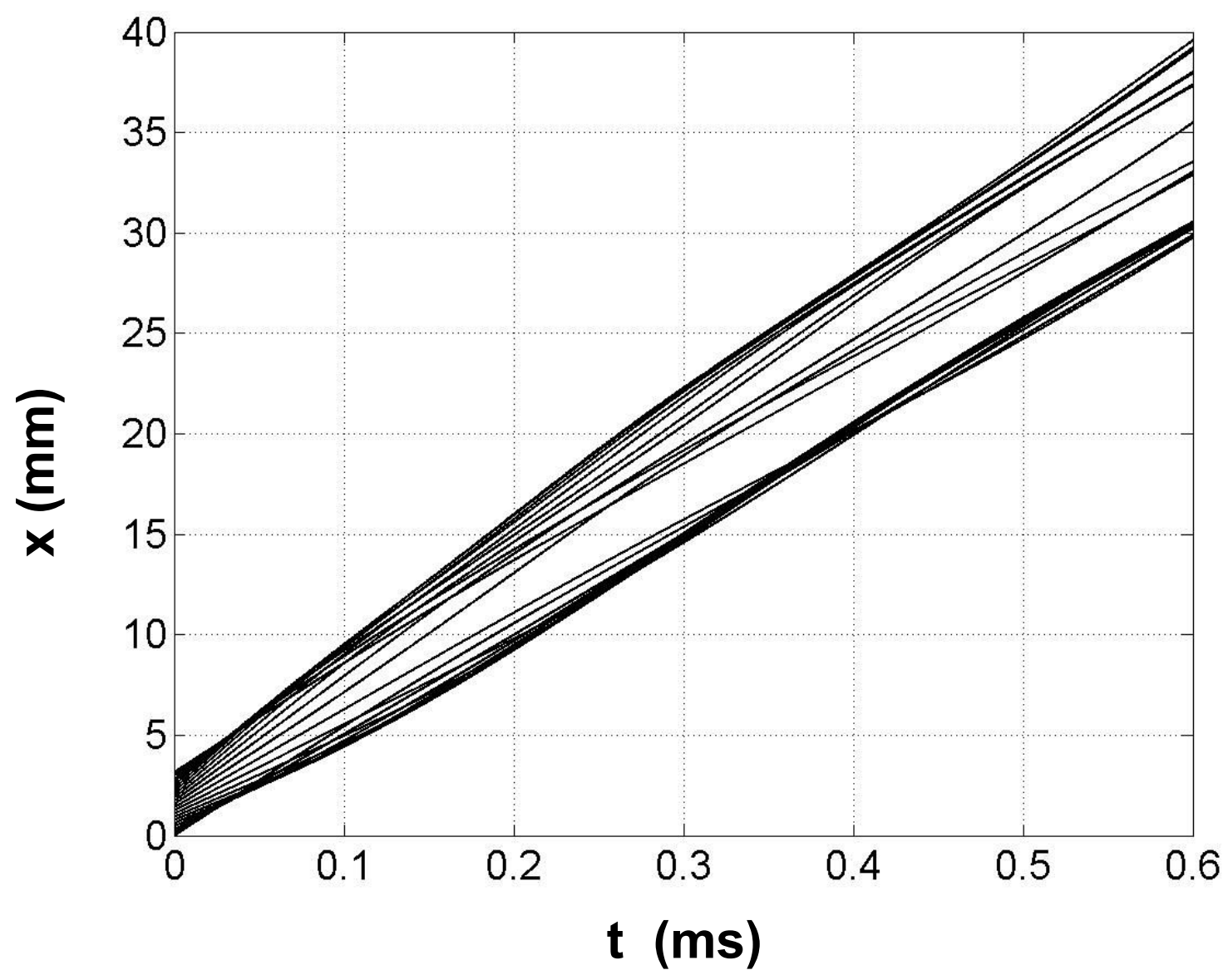

Fig. 9 


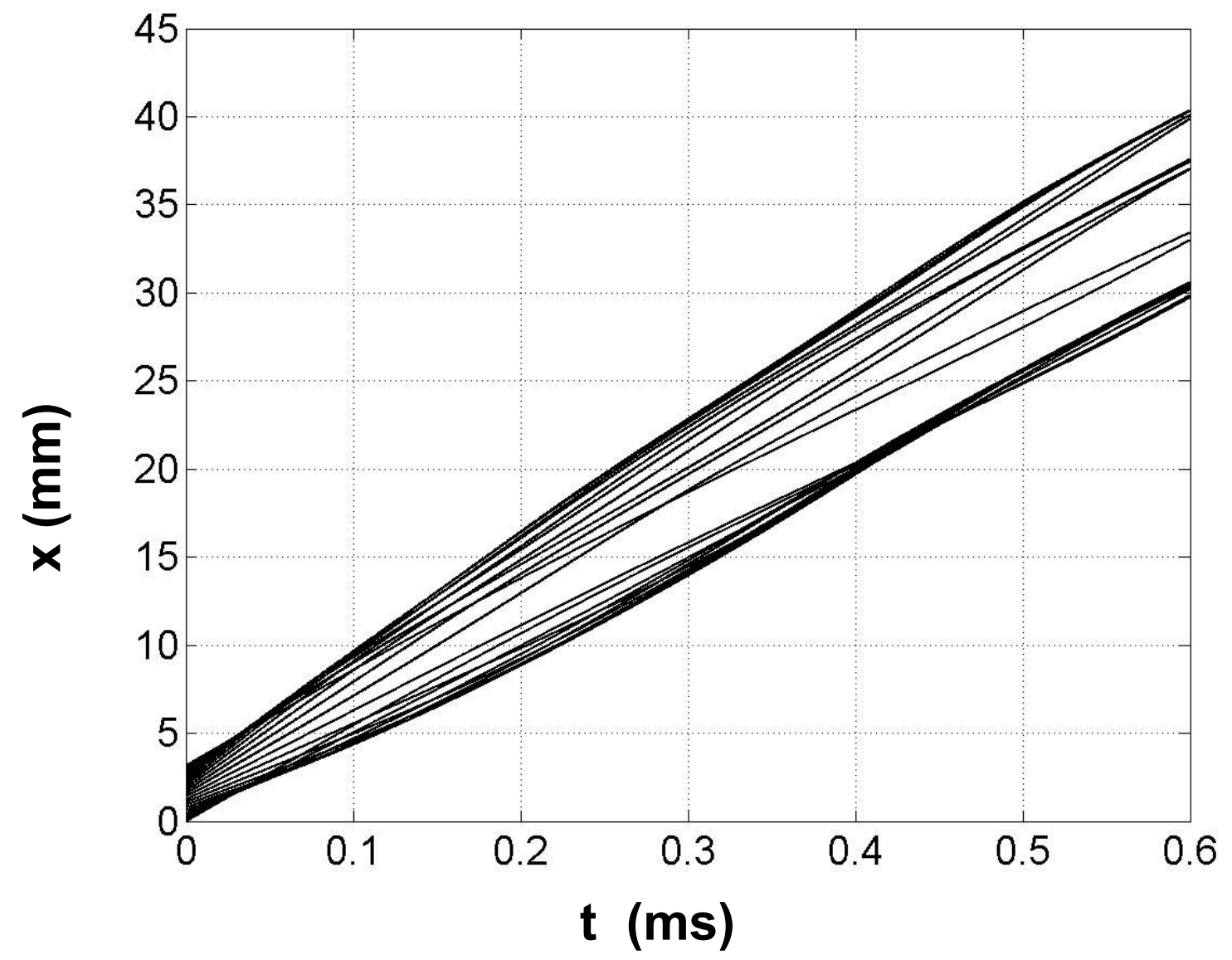

Fig. 10 


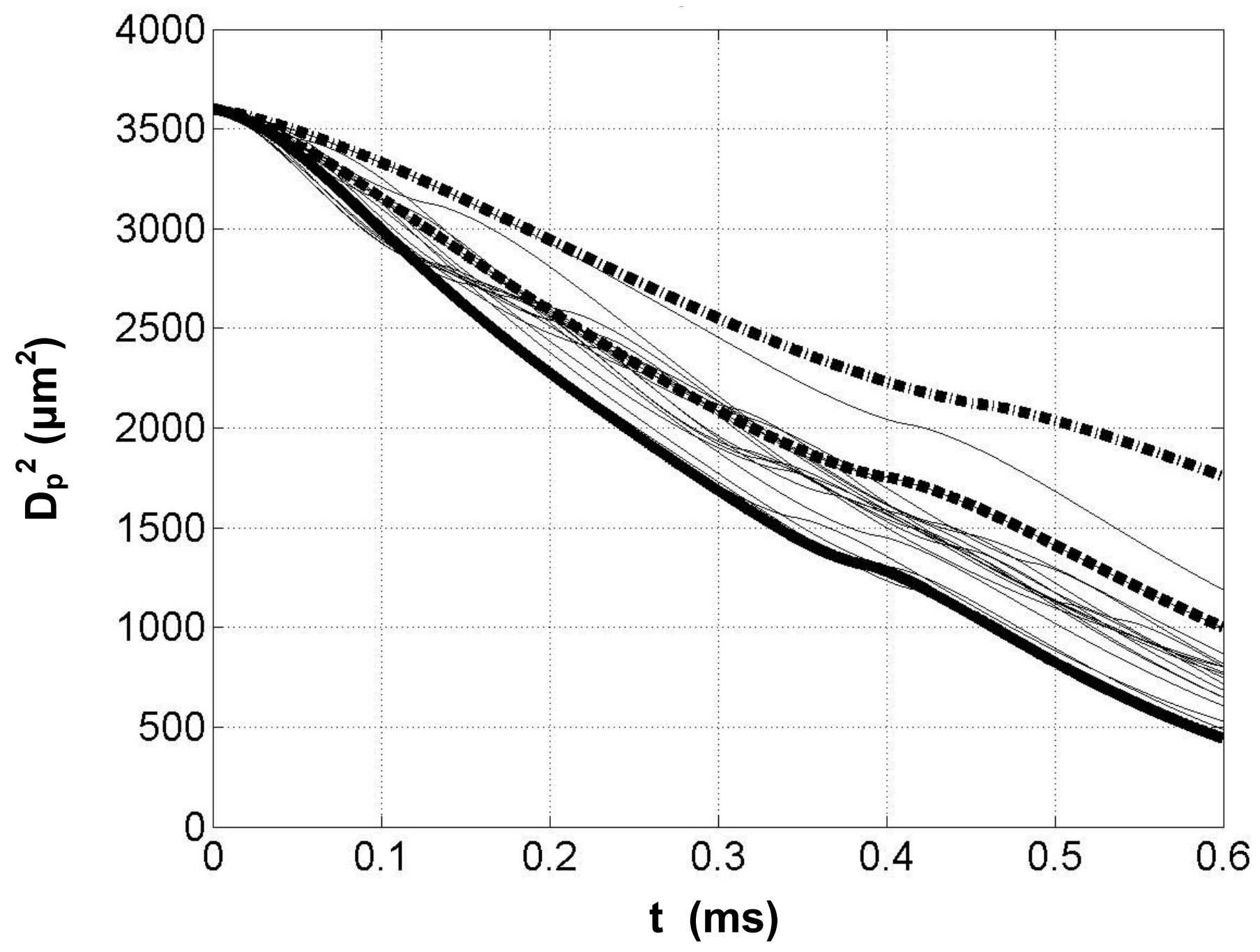

Fig. 11 


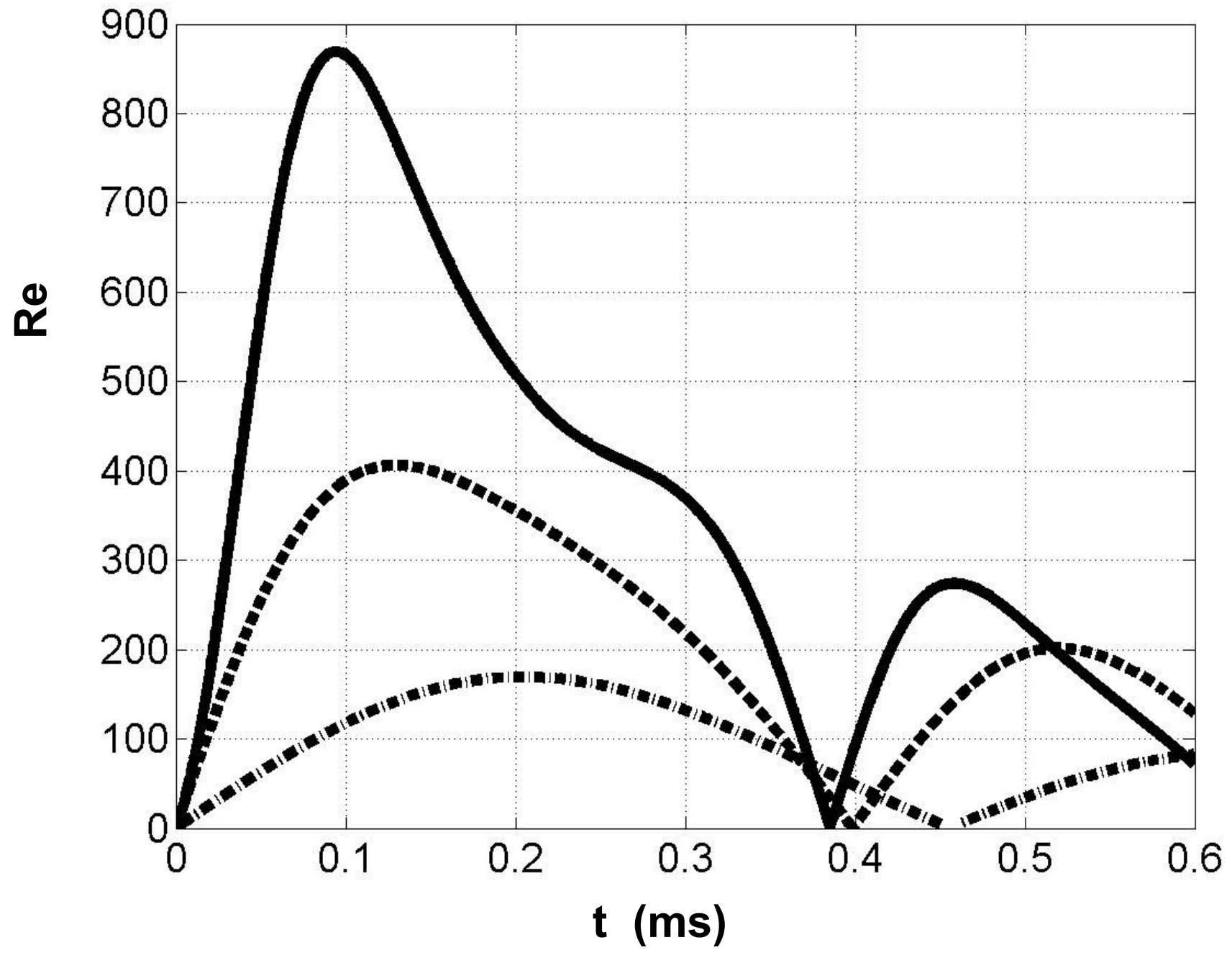

Fig. 12 


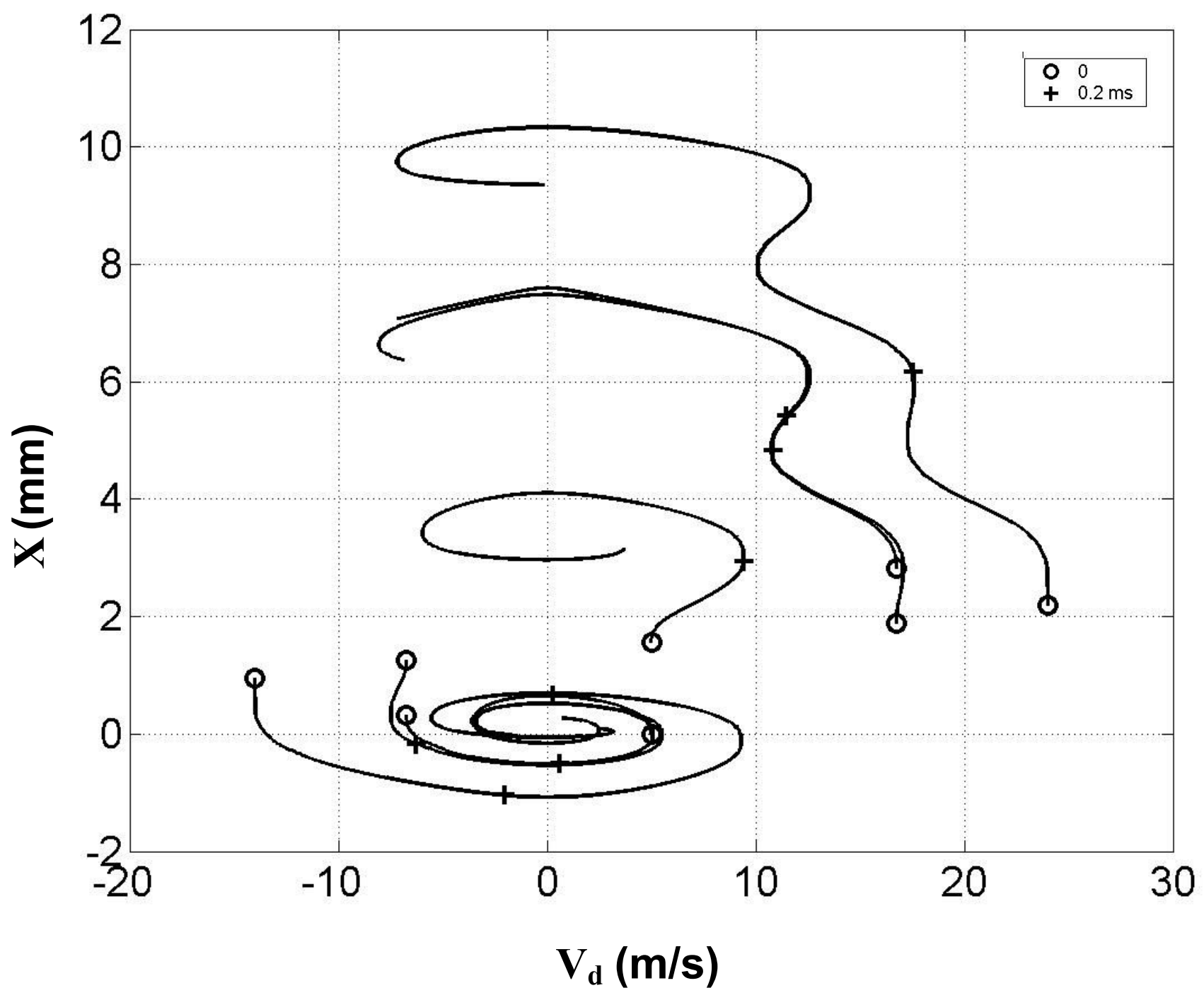

Fig. 13 
Ambient pressure

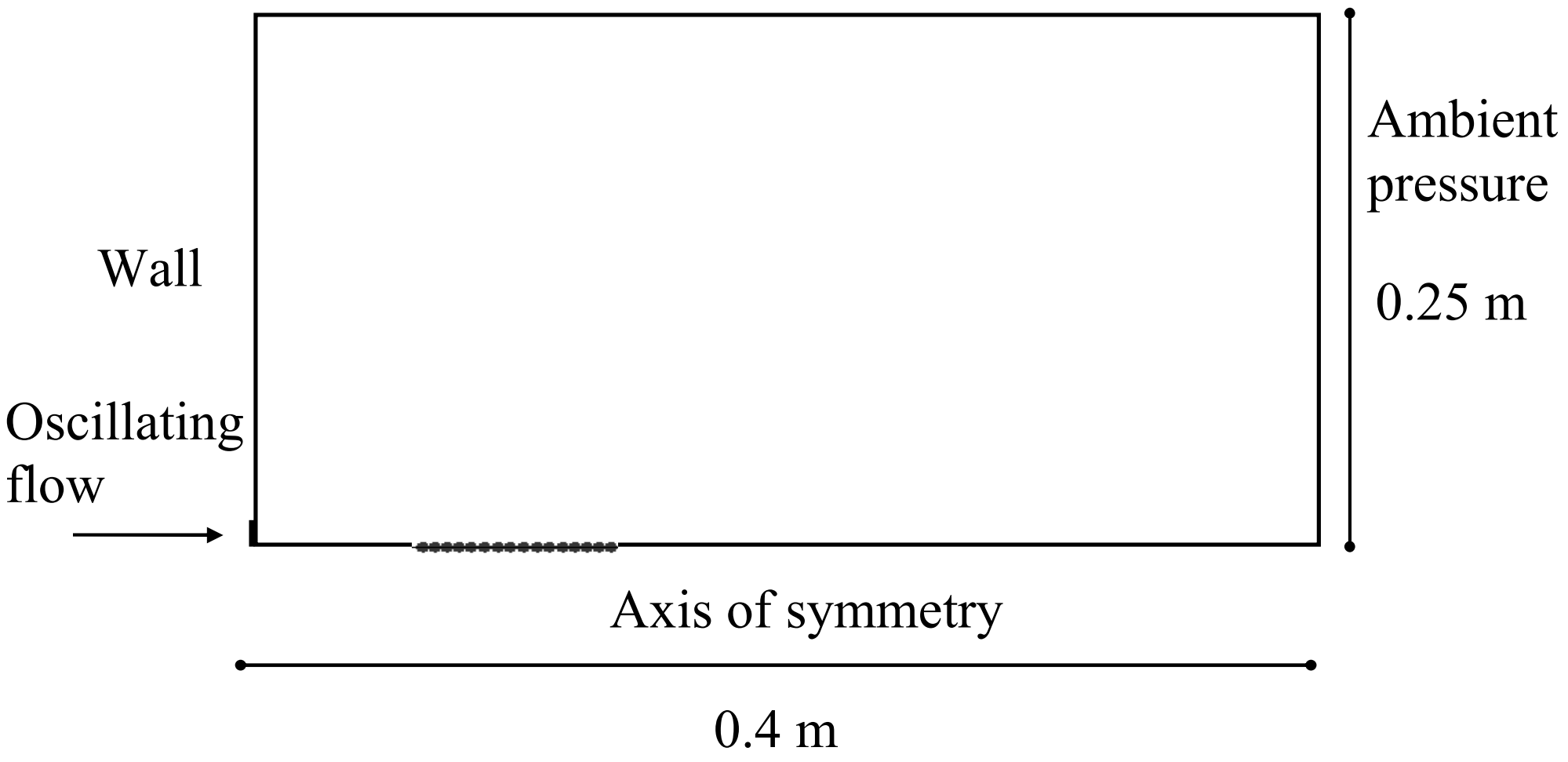

Fig. 14 

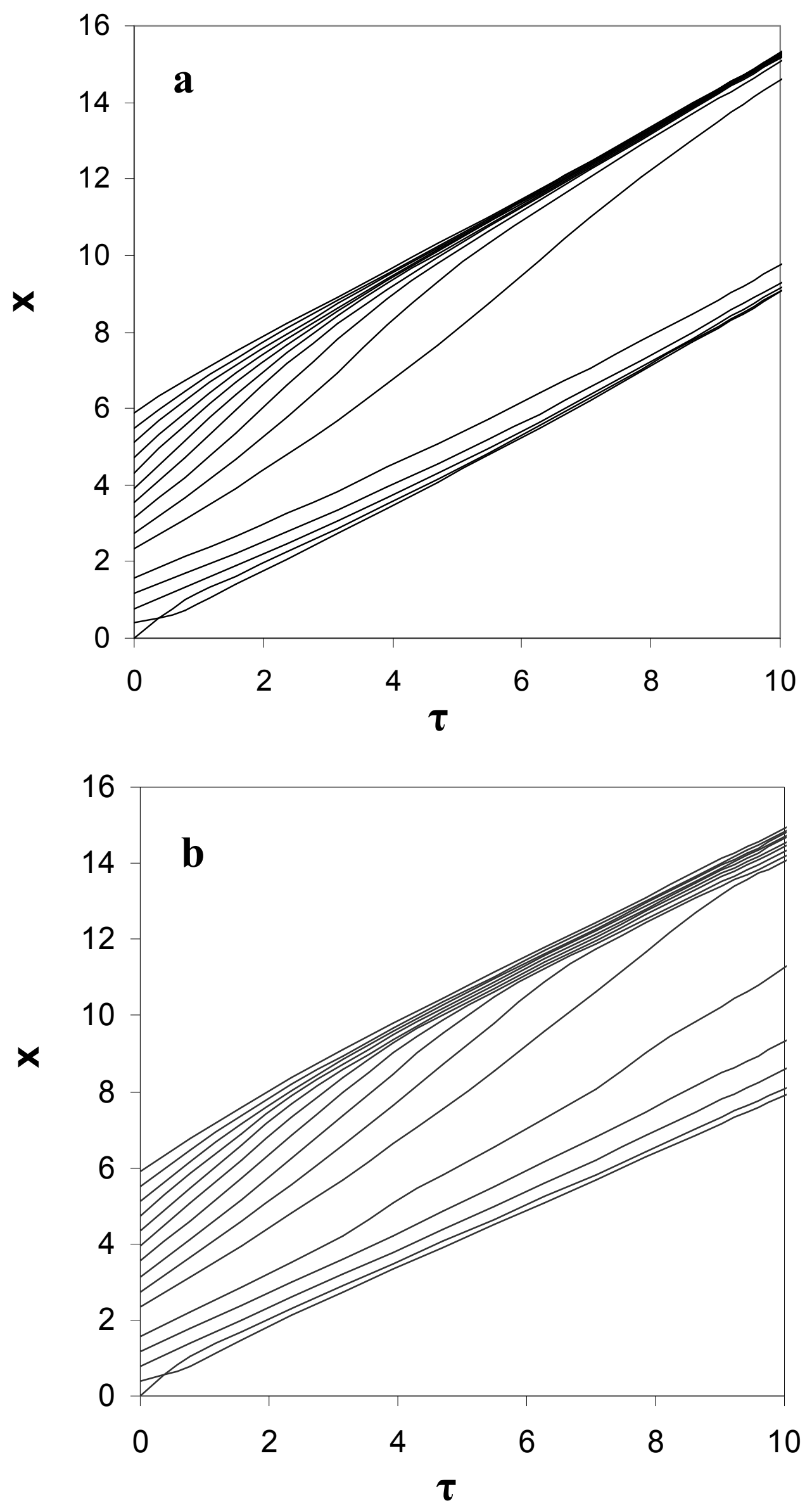


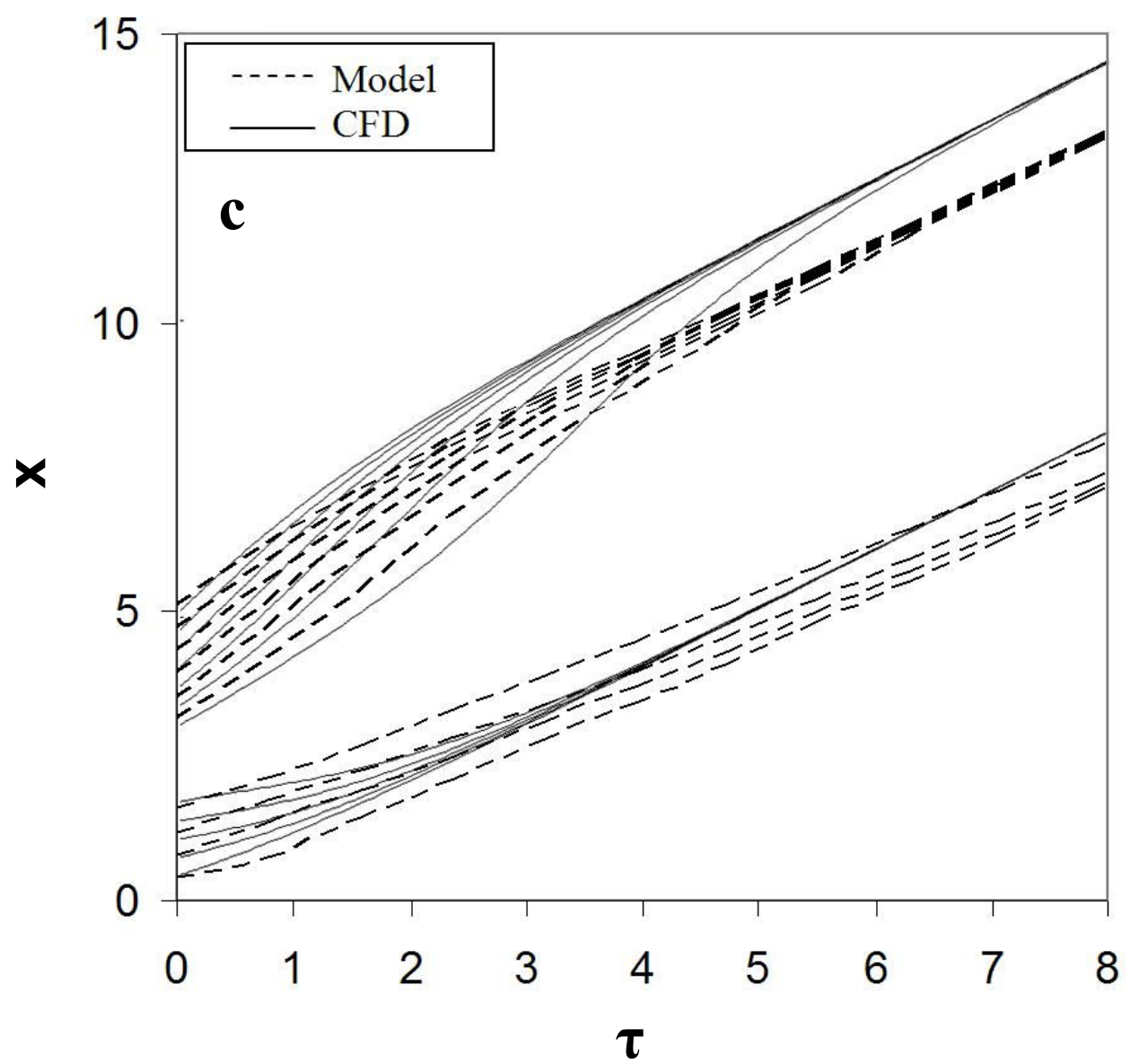

Fig. 15 


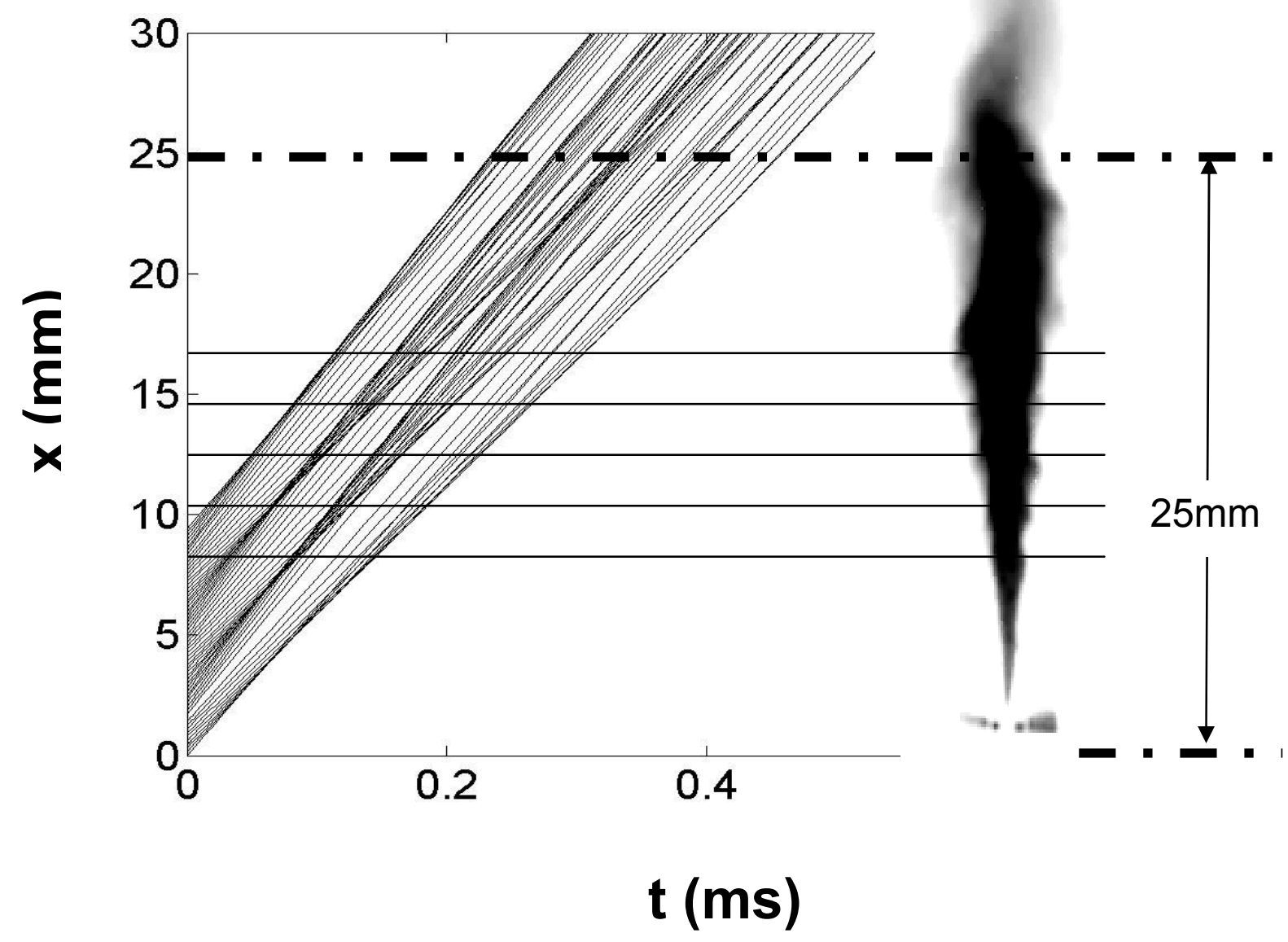

Fig. 16 


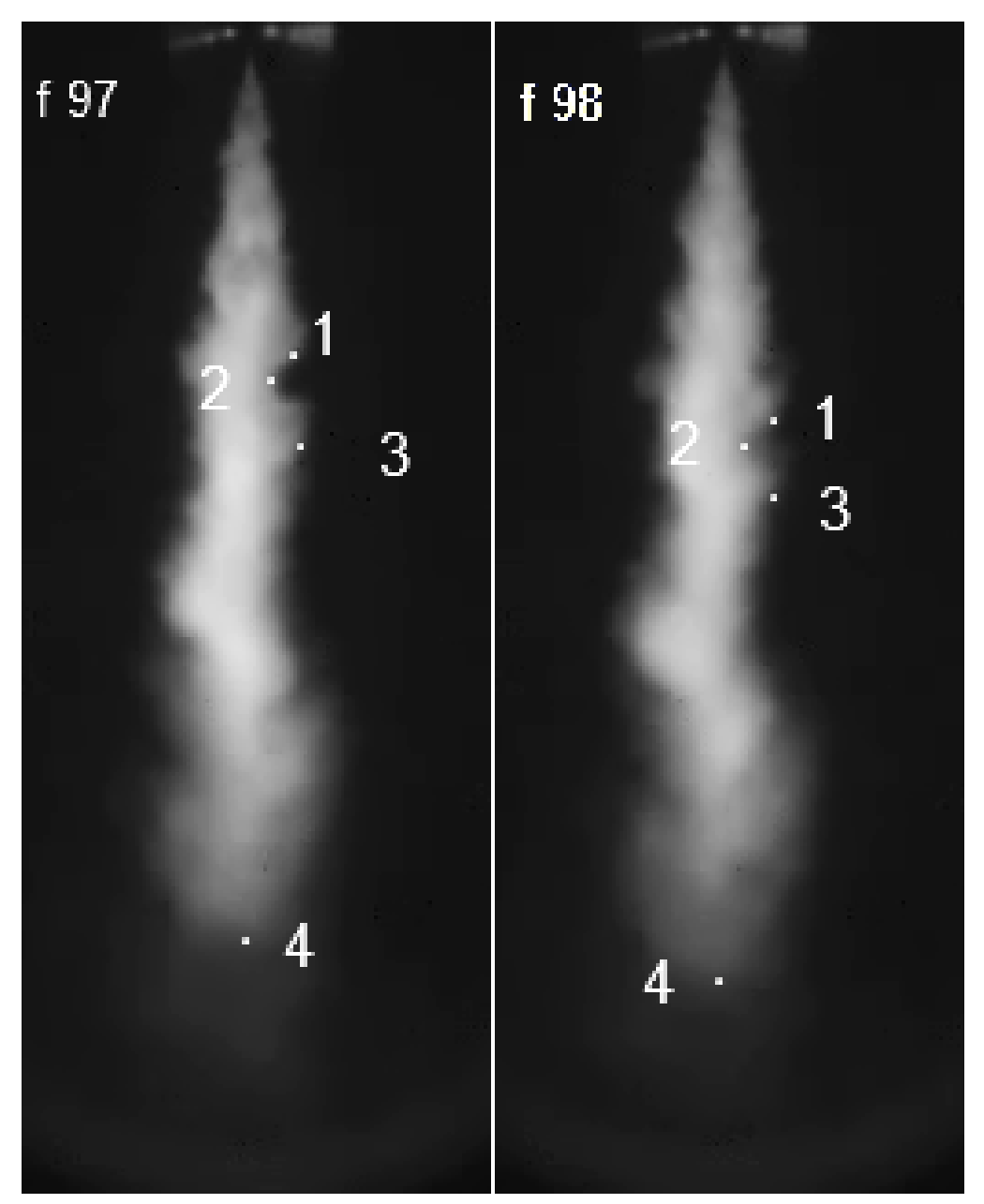

Fig. 17 